TRANSACTIONS OF THE

AMERICAN MATHEMATICAL SOCIETY

Volume 361, Number 9, September 2009, Pages 4593-4630

S 0002-9947(09)04576-0

Article electronically published on April 3, 2009

\title{
THE FUNDAMENTAL CROSSED MODULE OF THE COMPLEMENT OF A KNOTTED SURFACE
}

\author{
JOÃO FARIA MARTINS
}

\begin{abstract}
We prove that if $M$ is a CW-complex and $M^{1}$ is its 1-skeleton, then the crossed module $\Pi_{2}\left(M, M^{1}\right)$ depends only on the homotopy type of $M$ as a space, up to free products, in the category of crossed modules, with $\Pi_{2}\left(D^{2}, S^{1}\right)$. From this it follows that if $\mathcal{G}$ is a finite crossed module and $M$ is finite, then the number of crossed module morphisms $\Pi_{2}\left(M, M^{1}\right) \rightarrow \mathcal{G}$ can be re-scaled to a homotopy invariant $I_{\mathcal{G}}(M)$, depending only on the algebraic 2type of $M$. We describe an algorithm for calculating $\pi_{2}\left(M, M^{(1)}\right)$ as a crossed module over $\pi_{1}\left(M^{(1)}\right)$, in the case when $M$ is the complement of a knotted surface $\Sigma$ in $S^{4}$ and $M^{(1)}$ is the handlebody of a handle decomposition of $M$ made from its 0 - and 1-handles. Here, $\Sigma$ is presented by a knot with bands. This in particular gives us a geometric method for calculating the algebraic 2-type of the complement of a knotted surface from a hyperbolic splitting of it. We prove in addition that the invariant $I_{\mathcal{G}}$ yields a non-trivial invariant of knotted surfaces in $S^{4}$ with good properties with regard to explicit calculations.
\end{abstract}

\section{INTRODUCTION}

Let $(M, N)$ be a pair of based path-connected spaces. The concept of a crossed module arises from a universal description of the properties of the boundary map $\partial: \pi_{2}(M, N) \rightarrow \pi_{1}(N)$, together with the natural action of $\pi_{1}(N)$ on $\pi_{2}(M, N)$. These data define the crossed module $\Pi_{2}(M, N)$, called the "Fundamental Crossed Module of $(M, N)$ ".

Due to some strong theorems by J.H.C. Whitehead, it is possible, in principle, to calculate $\Pi_{2}\left(M, M^{1}\right)$ when $M$ is a connected CW-complex and $M^{1}$ is its 1-skeleton. The calculability of fundamental crossed modules is, in addition, enhanced by a 2dimensional van Kampen theorem due to R. Brown and P.J. Higgins, stating that, under mild conditions, the fundamental crossed module functor from the category of based pairs of path connected spaces to the category of crossed modules preserves colimits; see [7, 9, 5.

The crossed module $\Pi_{2}\left(M, M^{1}\right)$ determines not only $\pi_{1}(M)$ and $\pi_{2}(M)$ as a module over $\pi_{1}(M)$, but also it determines the $k$-invariant $k(M) \in H^{3}\left(\pi_{1}(M), \pi_{2}(M)\right)$;

Received by the editors June 18, 2007.

2000 Mathematics Subject Classification. Primary 57M05, 57Q45; Secondary 55Q20.

Key words and phrases. Knotted surfaces, crossed modules, homotopy 2-types.

This work had the financial support of FCT (Portugal), post-doctoral grant number SFRH/BPD/17552/2004, part of the research project POCI/MAT/60352/2004 ("Quantum Topology"), also financed by FCT.

(C)2009 American Mathematical Society Reverts to public domain 28 years from publication 
in other words all the algebraic 2-type of $M$. Thus it is a more powerful invariant than $\pi_{1}(M)$ and $\pi_{2}(M)$ alone.

Crossed modules admit an obvious notion of homotopy; see [47, 8, 20]. From the point of view of the homotopy type of a $\mathrm{CW}$-complex $M$, as a space, considering its fundamental crossed module $\Pi_{2}\left(M, M^{1}\right)$ introduces an ambiguity due to the choice of a cell decomposition. However, despite this asymmetry, the homotopy type of the fundamental crossed module $\Pi_{2}\left(M, M^{1}\right)$ depends only on the homotopy type of $M$ as a space; see [47, 8]. In fact this result can be improved. It is proved in this article that the crossed module $\Pi_{2}\left(M, M^{1}\right)$ does not depend on the CWdecomposition of $M$, up to free products, in the category of crossed modules, with $\Pi_{2}\left(D^{2}, S^{1}\right)$. Consequently, the fundamental crossed module $\Pi_{2}\left(M, M^{1}\right)$, up to free products with $\Pi_{2}\left(D^{2}, S^{1}\right)$, is a genuine homotopy invariant of $\mathrm{CW}$-complexes. This invariant determines the topological 2-type of $M$; see [1, 30.

In light of the current discussion, it is natural to consider crossed modules in Geometric Topology. In this article we present an algorithm for calculating the crossed module $\Pi_{2}\left(M, M^{(1)}\right)$ in the case when $M$ is the complement of a knotted surface $\Sigma$ in $S^{4}$ and $M^{(1)}$ is the 1-handlebody (made out of the 0- and 1-handles) of a handle decomposition of $M$. Both the handle decomposition of $M$ (following [22, 23, 12]) and the method for determining $\Pi_{2}\left(M, M^{(1)}\right)$ are defined from a hyperbolic splitting of $\Sigma$, or, more precisely, from a presentation of $\Sigma$ by a knot with bands. We thus have a completely geometric method for calculating the algebraic 2-type of the complement of a knotted surface from a hyperbolic splitting of it. This will be the main result of this article. Our method to determine the algebraic 2-type of the complement of a knotted surface in $S^{4}$ should be compared with Lomonaco's method in 31. See also 34.

We illustrate our algorithm by calculating $\Pi_{2}\left(M, M^{(1)}\right)$, where $M$ is the complement of a knotted surface, in some non-trivial cases. We use it for determining the second homotopy group of the Spun Trefoil complement, and we verify that the result agrees with previous calculations.

Given that the fundamental crossed module $\Pi_{2}\left(M, M^{1}\right)$ depends only on the homotopy type of the CW-complex $M$, as a space, up to free products with $\Pi_{2}\left(D^{2}, S^{1}\right)$, it follows that if $\mathcal{G}$ is a finite crossed module and $M$ is finite, then the number of crossed module morphisms from $\Pi_{2}\left(M, M^{1}\right)$ to $\mathcal{G}$ (which is finite) can be rescaled to a homotopy invariant $I_{\mathcal{G}}(M)$ (the "Crossed Module Invariant"). This again solves the problem that we introduced an ambiguity due to the choice of a CW-decomposition of $M$. This Crossed Module Invariant depends only on the topological 2-type of a space.

From the algorithm for calculating $\Pi_{2}\left(M, M^{(1)}\right)$ when $M$ is the complement of an embedded surface in $S^{4}$, a method follows for calculating $I_{\mathcal{G}}(M)$, where $\mathcal{G}$ is a finite crossed module. We prove that the Crossed Module Invariant $I_{\mathcal{G}}$ defines a nontrivial invariant of knotted surfaces, with good calculability properties, especially in the case of abelian crossed modules. We will give an example (appearing also in 19]) of a pair of knotted surfaces, each diffeomorphic to the disjoint union of two tori $S^{1} \times S^{1}$, with the same fundamental group of the complement, while distinguished by their crossed module invariants. An open problem is whether the Crossed Module Invariant is powerful enough to distinguish between knotted surfaces with the same fundamental group and the second homotopy group of the complement (seen as a $\pi_{1}$-module), but with distinct $k$-invariants. See also [38. 
In [17] we defined an invariant of knotted surfaces from any finite crossed module. The construction was inspired by previous work of D. Yetter and T. Porter on manifold invariants defined from algebraic models of homotopy 2-types (crossed modules of groups); see [48] and [39, 40]. This article should give, in particular, an interpretation of our previous construction.

\section{Contents}

Introduction 4593

1. Preliminaries and general results $\quad 4595$

1.1. Crossed modules $\quad 4595$

1.2. The significance of the fundamental crossed module $\Pi_{2}\left(M, M^{1}\right) \quad 4598$

2. Complements of knotted surfaces $\quad 4602$

2.1. Handle decompositions of complements of knotted surfaces 4603

2.2. The calculus 4609

2.3. Spun trefoil 4616

3. The "crossed module invariant" of homotopy types 4619

3.1. Definition of the invariant 4619

3.2. Applications to knotted surfaces 4621

Acknowledgements $\quad 4628$

References $\quad 4628$

\section{Preliminaries and general Results}

1.1. Crossed modules. Let $G$ and $E$ be two groups. A crossed module $\mathcal{G}=$ $(G, E, \partial, \triangleright)$ is given by a group morphism $\partial: E \rightarrow G$ and an action $\triangleright$ of $G$ on $E$ on the left by automorphisms. The conditions on $\triangleright$ and $\partial$ are:

(1) $\partial(X \triangleright e)=X \partial(e) X^{-1} ; \forall X \in G, \forall e \in E$,

(2) $\partial(e) \triangleright f=e f e^{-1} ; \forall e, f \in E$;

see for example [5, 10]. Notice that the second condition implies that $\operatorname{ker}(\partial)$ commutes with all $E$. Therefore, the action of $G$ on $E$ induces an action of coker $(\partial)$ on $\operatorname{ker}(\partial)$, by automorphisms. We call $G$ the base group and $E$ the principal group. A crossed module is called finite if both $G$ and $E$ are finite.

The significance of the definition of crossed modules for Geometric Topology stems from:

Example 1.1. Let $(M, N)$ be a pair of based path connected spaces. Then $\left(\pi_{1}(N), \pi_{2}(M, N), \partial, \triangleright\right) \doteq \Pi_{2}(M, N)$, where the boundary map $\partial: \pi_{2}(M, N) \rightarrow$ $\pi_{1}(N)$ and the action of $\pi_{1}(N)$ on $\pi_{2}(M, N)$ are the natural ones (see Figure 1), is a crossed module called the "Fundamental Crossed Module" of $(M, N)$. This is a result of J.H.C. Whitehead; see 45].

A modern proof of this result appears in [10]. 


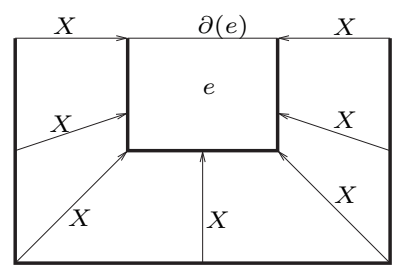

Figure 1. The action of an element $X \in \pi_{1}(N)$ on an $e \in \pi_{2}(M, N)$.

A morphism between the crossed modules $\mathcal{G}$ and $\mathcal{G}^{\prime}=\left(G^{\prime}, E^{\prime}, \partial^{\prime}, \triangleright^{\prime}\right)$ is given by a pair $(\phi, \psi)$ of group morphisms $\phi: G \rightarrow G^{\prime}$ and $\psi: E \rightarrow E^{\prime}$, making the diagram

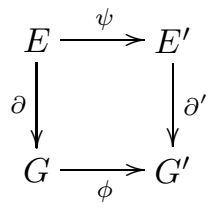

commutative.

In addition we must have

$$
\phi(X) \triangleright^{\prime} \psi(e)=\psi(X \triangleright e) ; \forall X \in G, \forall e \in E .
$$

Crossed modules and their morphisms form a category. This category is a category with colimits; see [10, 3.5] or [9].

Example 1.2. Let $(M, N, *)$ be a pair of based path connected spaces. If $*^{\prime} \in N$ is another base point and $\gamma$ is a path connecting $*$ to $*^{\prime}$, then there exists a natural isomorphism $\Pi_{2}(M, N, *) \rightarrow \Pi_{2}\left(M, N, *^{\prime}\right)$, where the maps on homotopy groups are the usual ones constructed from the path $\gamma$.

Example 1.3. Let $\mathcal{G}=(G, E, \partial, \triangleright)$ and $\mathcal{G}^{\prime}=\left(G^{\prime}, E^{\prime}, \partial^{\prime}, \triangleright^{\prime}\right)$ be crossed modules. The free product $\mathcal{G} \vee \mathcal{G}^{\prime}$ of $\mathcal{G}$ and $\mathcal{G}^{\prime}$ is the pushout, in the category of crossed modules, of the diagram

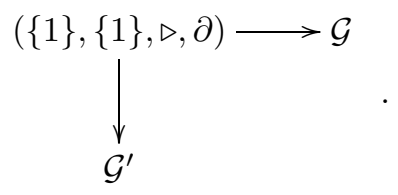

Recall that the category of crossed modules is a category with colimits.

Example 1.4. Let $M$ and $N$ be $\mathrm{CW}$-complexes with unique 0-cells, which we take to be their base points. We have

$$
\Pi_{2}\left(\left(M, M^{1}, *\right) \vee\left(N, N^{1}, *\right)\right) \cong \Pi_{2}\left(M, M^{1}, *\right) \vee \Pi_{2}\left(N, N^{1}, *\right) .
$$

This follows immediately from the (R. Brown and P.J. Higgins') 2-dimensional van Kampen Theorem stating that, under mild conditions, the crossed module functor preserves colimits, and in particular pushouts; see [5, 10, 7]. This is the case for the wedge product of two well pointed based spaces; see [9, Theorem 7.1]. 
Example 1.5. Let $\mathcal{G}=(G, E, \partial, \triangleright)$ be a crossed module. Suppose that the elements $a_{1}, \ldots, a_{n} \in E$ are such that $\partial\left(a_{k}\right)=1_{G}, k=1, \ldots, n$. Let $F$ be the subgroup of $E$ generated by the elements of the form $X \triangleright a_{k}$ where $X \in G, k=1, \ldots, n$; thus $F$ is normal in $E$ by the second condition of the definition of crossed modules. In fact $F$ commutes with all of $E$. Obviously both $\partial$ and the action of $G$ on $E$ descend to $E / F$. Denote the induced map and action by $\partial^{\prime}$ and $\triangleright^{\prime}$. It is easy to show that $\left(G, E / F, \partial^{\prime}, \triangleright^{\prime}\right)$ is a crossed module.

We will go back to this construction below.

1.1.1. Presentations of crossed modules. For details on free crossed modules see 10, 11, for example. Let $G$ be a group and $K$ be a set. The free crossed module $\mathcal{F}\left(\partial_{0}: K \rightarrow G\right)$ on a map $\partial_{0}: K \rightarrow G$ has $G$ as its base group. The principal group $E$ is the quotient of the free group on the set of pairs $(X, m)$, where $X \in G$ and $m \in K$, by the relations

$$
(X, m)(Y, n)(X, m)^{-1}=\left(X \partial_{0}(m) X^{-1} Y, n\right) ; X, Y \in G ; m, n \in K .
$$

The boundary map $\partial: E \rightarrow G$ is defined on generators by $\partial(X, m)=X \partial_{0}(m) X^{-1}$, whereas the action of $G$ on $E$ is given simply by $X \triangleright(Y, m)=(X Y, m)$; here $X, Y \in G$ and $m \in K$. Note that the map $\iota: K \rightarrow E$ such that $\iota(m)=\left(1_{G}, m\right)$ is injective. From now on we consider $K$ to be included in $E$ in this way.

The free crossed module $\mathcal{F}\left(\partial_{0}: K \rightarrow G\right)=(G, E, \partial, \triangleright)$ on a map $\partial_{0}: K \rightarrow G$ is defined, up to isomorphism, by the following natural universal property:

Lemma 1.6. Let $G^{\prime}$ be a group and let $\phi: G \rightarrow G^{\prime}$ be a morphism. Also let $\mathcal{G}^{\prime}=\left(G^{\prime}, E^{\prime}, \partial^{\prime}, \triangleright^{\prime}\right)$ be a crossed module. Consider a map $\psi_{0}: K \rightarrow E^{\prime}$ such that $\partial^{\prime} \circ \psi_{0}=\phi \circ \partial_{0}$. There exists a unique group morphism $\psi: E \rightarrow E^{\prime}$ extending $\psi_{0}$ in such a way that $(\phi, \psi)$ is a morphism of crossed modules $\mathcal{G} \rightarrow \mathcal{G}^{\prime}$.

Let $G$ be a group and $K$ be a set. Also let $\partial_{0}: K \rightarrow G$ be a map. Consider the free crossed module $\mathcal{F}\left(\partial_{0}: K \rightarrow G\right)=(G, E, \partial, \triangleright)$. A 2-relation is, by definition, an element $r$ of $E$ with $\partial(r)=1_{G}$.

Let $R=\left\{r_{1}, \ldots, r_{n}\right\}$ be a set of 2-relations, which we can take to be infinite. The crossed module presented by the map $\partial_{0}: K \rightarrow G$, with 2-relations $\left\{r_{1}, \ldots, r_{n}\right\}$, say $\mathcal{U}\left(\partial_{0}: K \rightarrow G ; r_{1}, \ldots, r_{n}=1\right)$, is, by definition, the crossed module constructed from $\mathcal{F}\left(\partial_{0}: K \rightarrow G\right)$ and $\left\{r_{1}, \ldots, r_{n}\right\}$ as in Example 1.5.

1.1.2. A decomposition of certain free crossed modules. This section will only be used for the explicit calculation of the second homotopy groups of the Spun Trefoil and the Spun Hopf Link complements.

Let $G$ be some group. Let $K$ be a set provided with a map $\partial_{0}: K \rightarrow G$. Suppose that $K$ is the disjoint union of the sets $K_{1}$ and $K_{2}$. Let $\partial_{0}^{1}$ and $\partial_{0}^{2}$ be the restrictions of $\partial_{0}$ to $K_{1}$ and $K_{2}$, respectively. Suppose also that $\partial_{0}^{2}(k)=1_{G}, \forall k \in K_{2}$. Let $F_{2}$ be the subgroup of the principal group of $\mathcal{F}\left(\partial_{0}: K \rightarrow G\right)=(G, E, \partial, \triangleright)$ generated by the elements $X \triangleright f$, where $f \in K_{2}$ and $X \in G$. Then it is easy to see that $F_{2}$ commutes with $E$ and that it is the free abelian module over $\mathbb{Z}(\operatorname{coker}(\partial))$ with base $K_{2}$, with the obvious action of $G$. This follows from relations (1.1) and the fact that for any crossed module $\left(G^{\prime}, E^{\prime}, \partial^{\prime}, \triangleright^{\prime}\right)$, the image $\operatorname{im}\left(\partial^{\prime}\right)$ of $\partial^{\prime}$ acts trivially on $\operatorname{ker}\left(\partial^{\prime}\right)$ - a consequence of the second condition of the definition of crossed modules.

In fact, the group $F_{2}$ has an algebraic complementary $F_{1}$ in $E$, as a group and as a $G$-module, where $F_{1}$ is isomorphic with the principal group of the free crossed module on the map $\partial_{0}^{1}: K_{1} \rightarrow G$. This follows immediately from relations (1.1). 
Therefore we have:

Proposition 1.7. Let $G$ be a group, and let $K$ be a set provided with a map $\partial_{0}: K \rightarrow G$. Suppose that $K$ is the disjoint union of the sets $K_{1}$ and $K_{2}$. Let $\partial_{0}^{1}$ and $\partial_{0}^{2}$ be the restrictions of $\partial_{0}$ to $K_{1}$ and $K_{2}$, respectively. Suppose also that $\partial_{0}^{2}(k)=1_{G}, \forall k \in K_{2}$. Then as a $G$-module and as a group, the principal group of the free crossed module $(G, E, \partial, \triangleright)$ on $\partial_{0}: K \rightarrow G$ is the direct sum of the principal group $F_{1}$ of the free crossed module on $\partial_{0}^{1}: K_{1} \rightarrow G$ and the free $\mathbb{Z}(\operatorname{coker}(\partial))$-module $F_{2}$ with base $K_{2}$, with the obvious action of $G$. This direct sum has the natural boundary map to $G$ where $\partial\left(F_{2}\right)=\left\{1_{G}\right\}$.

This result is also valid, with the obvious modifications, for the case of a crossed module $\mathcal{U}\left(\partial_{0}: K \rightarrow G ; r_{1}, \ldots, r_{n}=1\right)$ presented by a map $\partial_{0}: K \rightarrow G$ with 2relations $r_{1}, \ldots, r_{n}$, as long as each 2-relation is contained in either $F_{1}$ or $F_{2}$.

1.2. The significance of the fundamental crossed module $\Pi_{2}\left(M, M^{1}\right)$. Whenever $E$ is an abelian group and we have a left action of $G$ on $E$ by automorphisms, then $\left(G, E, \partial=1_{G}, \triangleright\right)$ is always a crossed module. For any based path connected topological space $M$, the group $\pi_{2}(M)$ is abelian and $\pi_{1}(M)$ acts on $\pi_{2}(M)$ by automorphisms. Therefore we have a crossed module $\pi_{1,2}(M)$ for any based topological space. See for example [31, 34 for calculations of $\pi_{1,2}(M)$ when $M$ is the complement of a knotted surface in $S^{4}$.

A first idea about how to employ the notion of a crossed module to define invariants of manifolds could be to consider the crossed module $\pi_{1,2}(M)$. However, even when $M$ is a compact manifold, it is not certain that $\pi_{2}(M)$ is finitely generated as a module over $\pi_{1}(M)$; see [31, problem 5] and, less directly related, 31, problems 6 and 13] for the important case of complements of knotted surfaces. Therefore, $\pi_{1,2}(M)$ is not a very practical invariant since it not easy to distinguish between two non-finitely generated $\pi_{1}(M)$-modules.

Another solution is to consider the more tractable relative case. Let $M$ be a CWcomplex, and let $M^{1}$ be its 1-skeleton. Consider the crossed module $\Pi_{2}\left(M, M^{1}\right)=$ $\left(\pi_{1}\left(M^{1}\right), \pi_{2}\left(M, M^{1}\right), \partial, \triangleright\right)$. Despite the asymmetry introduced by choosing a particular CW-decomposition of $M$, this crossed module determines $\pi_{2}(M)$ and $\pi_{1}(M)$, which fit inside the exact sequence

$$
\{0\} \rightarrow \pi_{2}(M) \rightarrow \pi_{2}\left(M, M^{1}\right) \stackrel{\partial}{\rightarrow} \pi_{1}\left(M^{1}\right) \rightarrow \pi_{1}(M) \rightarrow\{1\},
$$

since $\pi_{2}\left(M^{1}\right)=\{0\}$. In fact the crossed module $\Pi_{2}\left(M, M^{1}\right)$ also determines the $k$-invariant $k(M) \in H^{3}\left(\pi_{1}(M), \pi_{2}(M)\right)$. The group cohomology class $k(M)$ is determined from the classical correspondence between 3-dimensional group cohomology classes and crossed modules; see for example [5, 32, 3, 26. Therefore the crossed module $\Pi_{2}\left(M, M^{1}\right)$ determines the topological 2-type of $M$ (see [33]), and thus it is strictly stronger than $\pi_{1}(M)$ and $\pi_{2}(M)$ alone (see [1, 30]).

Choosing the apparently less charming relative case is also justified by the fact that $\Pi_{2}\left(M, M^{1}\right)$ does not depend on the cellular decomposition of $M$ up to free products, in the category of crossed modules, with $\Pi_{2}\left(D^{2}, S^{1}\right)$, as we will prove below in Section 1.2.2 This expands an old result of J.H.C. Whitehead stating that the homotopy type of the crossed module $\Pi_{2}\left(M, M^{1}\right)$ depends only on the homotopy type of $M$, as a space; see [47, 8]. 
Therefore the fundamental crossed module $\Pi_{2}\left(M, M^{1}\right)$, up to free products with $\Pi_{2}\left(D^{2}, S^{1}\right)$, is a genuine homotopy invariant. This allows us to obtain a homotopy invariant of finite connected CW-complexes for any finite crossed module, see Section 3.1 .

We will see below that in the case when $M$ is a CW-complex and $M^{1}$ is its 1-skeleton, then $\Pi_{2}\left(M, M^{1}\right)$ is, in principle, calculable.

1.2.1. Whitehead's isomorphisms. For a more complete treatment of these issues see [2]. Let $N$ be a path-connected topological space with a base point $*$. Let $M$ be a topological space obtained from $N$ by attaching some 2-cells (or 2-handles) $s_{1}, \ldots, s_{n}$. Choose a base point $*_{i}$ on the boundary of each 2 -cell, where $i=1, \ldots, n$. If we are provided paths connecting $*_{i}$ with $*$, for $i=1, \ldots, n$, then each 2 -cell $s_{i}$ can be identified uniquely with an element of $\pi_{2}(M, N, *)$. This does not depend on the path chosen up to acting by some element of $\pi_{1}(N, *)$.

Consider the map $\partial_{0}:\left\{s_{1}, \ldots, s_{n}\right\} \rightarrow \pi_{1}(N, *)$ induced by the attaching maps of each cell $s_{i}$. This map (well defined up to conjugations by elements of $\pi_{1}(N, *)$ ) is also fixed by the chosen paths connecting $*_{i}$ with $*$, for $i=1, \ldots, n$.

Recall the following theorem, due to J.H.C. Whitehead. For the original proof see [45, 46, 47]; see also [10, 5.4] and [6, 8, 24].

Theorem 1.8. The natural morphism from the free crossed module on the map $\partial_{0}:\left\{s_{1}, \ldots, s_{n}\right\} \rightarrow \pi_{1}(N, *)$ into $\Pi_{2}(M, N, *)$ is an isomorphism of crossed modules.

This result (usually called Whitehead's Theorem) is one of the most important results that we will use.

Let $M$ be a $\mathrm{CW}$-complex. For each $k \in \mathbb{N}_{0}$, let $M^{k}$ denote the $k$-skeleton of $M$. For simplicity, suppose that $M$ has a unique 0 -cell, which we take to be its base point $*$. Note that from the Cellular Approximation Theorem we have $\Pi_{2}\left(M, M^{1}, *\right) \cong \Pi_{2}\left(M^{3}, M^{1}, *\right)$.

Consider the group complex

$$
\ldots \stackrel{\partial_{4}}{\longrightarrow} \pi_{3}\left(M^{3}, M^{2}, *\right) \stackrel{\partial_{3}}{\longrightarrow} \pi_{2}\left(M^{2}, M^{1}, *\right) \stackrel{\partial_{2}}{\longrightarrow} \pi_{1}\left(M^{1}, *\right) \stackrel{p}{\rightarrow} \pi_{1}(M, *)
$$

with the obvious boundary maps. This is a crossed complex of free type, called the "Fundamental Crossed Complex of $M$ "; see for example [5, 6. In particular, we have an action of the group $\pi_{1}\left(M^{1}, *\right)$ on all the other groups, preserving the boundary maps, and such that, if $n>2$, then the action of $\pi_{1}\left(M^{1}, *\right)$ on $\pi_{n}\left(M^{n}, M^{n-1}, *\right)$ factors through the projection map $p: \pi_{1}\left(M^{1}, *\right) \rightarrow \pi_{1}(M, *)$.

For each $n$-cell $c^{n}$ of $M$, where $n>1$, choose a base point on the boundary of it, as well as a path from the cell base point to $*$. Therefore $c^{n}$ determines an element $c^{n} \in \pi_{n}\left(M^{n}, M^{n-1}, *\right)$, and its attaching map can also be identified with an element of $\pi_{n-1}\left(M^{n-1}, *\right)$. If $n>2$, the projection of this element in $\pi_{n-1}\left(M_{n-1}, M_{n-2}, *\right)$ is the boundary map $\partial_{n}\left(c^{n}\right) \in \pi_{n-1}\left(M_{n-1}, M_{n-2}, *\right)$ of $c^{n}$, considering the fundamental complex complex of $M$.

It is well known (see [44, V.1], [47] or [5]) that, if $n>2$, then the natural map from the free $\mathbb{Z}\left(\pi_{1}(M, *)\right)$-module over the $n$-cells of $M$ into $\pi_{n}\left(M^{n}, M^{n-1}, *\right)$ is an isomorphism. This is also a result of J.H.C. Whitehead. In particular, the group $\pi_{3}\left(M^{3}, M^{2}, *\right)$ is the free $\mathbb{Z}\left(\pi_{1}\left(M^{2}, *\right)\right)$-module on the group elements defined by the 3-cells of $M$. 
From the long homotopy exact sequence of the triple $\left(M^{3}, M^{2}, M^{1}\right)$ it follows that:

\section{Lemma 1.9.}

$$
\pi_{2}\left(M, M^{1}, *\right)=\pi_{2}\left(M^{2}, M^{1}, *\right) / \operatorname{im}\left(\partial_{3}\right) .
$$

Let $c_{1}^{3}, \ldots, c_{n_{3}}^{3}$ be the 3 -cells of $M$. Each one of them defines an element of $\pi_{3}\left(M^{3}, M^{2}, *\right)$, as well as its boundary $\partial_{3}\left(c_{k}^{3}\right) \in \pi_{2}\left(M^{2}, M^{1}, *\right), k=1, \ldots, n_{3}$, well defined if we make the choices above. Also let $\left\{c_{1}^{2}, \ldots, c_{n_{2}}^{2}\right\}$ be the set of 2-cells of $M$, where $c_{k}^{2}$ attaches along $\partial_{2}\left(c_{k}^{2}\right) \in \pi_{1}\left(M^{1}, *\right), k=1, \ldots, n_{2}$.

Theorem 1.10. The crossed module $\Pi_{2}\left(M, M^{1}, *\right)$ is the crossed module presented by the map from the set of 2-cells of $M$ into $\pi_{1}\left(M^{1}, *\right)$, defined from the attaching maps of each 2-cell of $M$, with one 2-relation for each 3-cell of $M$. More precisely:

$$
\Pi_{2}\left(M, M^{1}, *\right) \cong \mathcal{U}\left(\left\{c_{1}^{2}, \ldots, c_{n_{2}}^{2}\right\} \stackrel{\partial_{2}}{\longrightarrow} \pi_{1}\left(M^{1}, *\right) ; \partial_{3}\left(c_{1}^{3}\right), \ldots, \partial_{3}\left(c_{n_{3}}^{3}\right)=1\right) .
$$

Note that $\partial_{2} \circ \partial_{3}=1$.

Proof. By Whitehead's Theorem and the previous lemma, we only need to prove that $\operatorname{im}\left(\partial_{3}\right)$ is the subgroup of $\pi_{2}\left(M^{2}, M^{1}, *\right)$ generated by the elements $X \triangleright \partial_{3}\left(c_{i}^{3}\right)$, where $X \in \pi_{1}\left(M^{1}, *\right)$ and $i=1, \ldots, n_{3}$. This follows from the fact that $\pi_{3}\left(M^{3}, M^{2}, *\right)$ is the free $\mathbb{Z}\left(\pi_{1}\left(M^{2}, *\right)\right)$-module on the set of 3-cells of $M$.

This theorem tells us that, in principle, if $M$ is a $\mathrm{CW}$-complex and $*$ is a 0 -cell of $M$, then the crossed module $\Pi_{2}\left(M, M^{1}, *\right)$ can be calculated. The only possible difficulty is the determination of the boundary maps in the fundamental crossed complex of $M$. This can be solved for example for simplicial complexes by the homotopy addition lemma in [44, page 175]; see [20. The case of complements of knotted surfaces can also be solved by using a particular handle decomposition of them. This is the main aim of this article.

1.2.2. The dependence of $\Pi_{2}\left(M, M^{1}\right)$ on the cell decomposition of the $C W$-complex $M$. Let $(M, N)$ be a pair of connected CW-complexes such that the inclusion of $N$ in $M$ is a homotopy equivalence. Let $N^{1}$ and $M^{1}$ be, respectively, the 1-skeletons of $N$ and $M$. Suppose that $N$ has a unique 0 -cell, which we take to be the base point $*$ of $N$ and $M$, so that both $N$ and $M$ are well pointed.

The group $\pi_{1}\left(N^{1}, *\right)$ is the free group on the set $\left\{d_{1}, \ldots, d_{n}\right\}$ of 1-cells of $N$. There also exist $c_{1}, \ldots, c_{m} \in \pi_{1}\left(M^{1}, *\right)$ such that $\pi_{1}\left(M^{1}, *\right)$ is the free group $F\left(d_{1}, \ldots, d_{n}, c_{1}, \ldots, c_{m}\right)$ on the set $\left\{d_{1}, \ldots, d_{n}, c_{1}, \ldots, c_{m}\right\}$. Note that these elements of $\pi_{1}\left(M^{1}, *\right)$ define elements of $\pi_{1}(M, *)$ in the obvious way.

Theorem 1.11. There exists a homotopy equivalence:

$$
\left(M, M^{1}, *\right) \cong\left(N, N^{1}, *\right) \vee\left(D^{2}, S^{1}, *\right)^{\vee m} .
$$

Proof 1 Since $N$ is a subcomplex of $M$, and $M$ is homotopic to $N$, it follows that $N$ is a strong deformation retract of $M$. By the Cellular Approximation Theorem, we can suppose, further, that there exists a retraction $r: M \rightarrow N$ sending $M^{1}$ to

\footnotetext{
${ }^{1}$ This argument arose in a discussion with Gustavo Granja.
} 
$N^{1}$, and such that $r \cong \operatorname{id}_{M}$, relative to $N$. In particular, if $k \in\{1, \ldots, m\}$, then we have that $c_{k} r_{*}\left(c_{k}\right)^{-1}=1_{\pi_{1}(M, *)}$, in $\pi_{1}(M, *)$. Define a map

$$
f:\left(P, P^{1}, *\right) \doteq\left(N, N^{1}, *\right) \vee \bigvee_{k=1}^{m}\left(D_{k}^{2}, S_{k}^{1}, *\right) \rightarrow\left(M, M^{1}, *\right)
$$

in the following way.

First of all, send $\left(N, N^{1}, *\right)$ identically to its copy $\left(N, N^{1}, *\right) \subset\left(M, M^{1}, *\right)$. Then we send each $S_{k}^{1}$ to the element $c_{k} r_{*}\left(c_{k}\right)^{-1} \in \pi_{1}\left(M^{1}, *\right)$. Since these elements are null homotopic in $(M, *)$, this map extends to the remaining 2-cells of $\left(P, P^{1}, *\right)$.

Let us prove that $f:\left(P, P^{1}, *\right) \rightarrow\left(M, M^{1}, *\right)$ is a homotopy equivalence. It suffices to prove that $f:(P, *) \rightarrow(M, *)$ and $f^{1} \doteq f_{\mid P^{1}}:\left(P^{1}, *\right) \rightarrow\left(M^{1} . *\right)$ are based homotopy equivalences, since all inclusion maps are cofibrations; see for example [35. 6.5]. Notice that the result proved there is also valid in the base case, as long as all the spaces considered are well pointed, which is the case here.

It is immediate that $f$ is an equivalence of homotopy $(P, *) \rightarrow(M, *)$, since $f$ extends the inclusion map $(N, *) \rightarrow(M, *)$. Let us now show that the map $f^{1}:\left(P^{1}, *\right) \rightarrow\left(M^{1}, *\right)$ is a homotopy equivalence. It is enough to prove that the induced map $f_{*}^{1}: \pi_{1}\left(P^{1}, *\right) \rightarrow \pi_{1}\left(M^{1}, *\right)$ is an isomorphism. Note that the group $\pi_{1}\left(P^{1}, *\right)$ is (similarly with $\pi_{1}\left(M^{1}, *\right)$ ) isomorphic with the free group on the set $\left\{d_{1}, \ldots, d_{n}, c_{1}, \ldots, c_{m}\right\}$. The induced map on the fundamental groups has the form

$$
f_{*}^{1}\left(d_{k}\right)=d_{k}, k=1, \ldots, n \text {, and } f_{*}^{1}\left(c_{k}\right)=c_{k} r_{*}\left(c_{k}\right)^{-1}, k=1, \ldots, m .
$$

Notice that $r_{*}\left(c_{k}\right) \in F\left(d_{1}, \ldots, d_{n}\right), k=1, \ldots, m$. Consider the morphism $g$ of $F\left(d_{1}, \ldots, d_{n}, c_{1}, \ldots, c_{m}\right)$ on itself such that

$$
g\left(d_{k}\right)=d_{k}, k=1, \ldots, n, \text { and } g\left(c_{k}\right)=c_{k} r_{*}\left(c_{k}\right), k=1, \ldots, m .
$$

Therefore $\left(f_{*}^{1} \circ g\right)\left(d_{k}\right)=d_{k}, k=1, \ldots, n$, and

$$
\begin{aligned}
\left(f_{*}^{1} \circ g\right)\left(c_{k}\right) & =f_{*}^{1}\left(c_{k} r_{*}\left(c_{k}\right)\right) \\
& =f_{*}^{1}\left(c_{k}\right) f_{*}^{1}\left(r_{*}\left(c_{k}\right)\right) \\
& =c_{k} r_{*}\left(c_{k}\right)^{-1} r_{*}\left(c_{k}\right) \\
& =c_{k}, k=1, \ldots, m .
\end{aligned}
$$

On the other hand $\left(g \circ f_{*}^{1}\right)\left(d_{k}\right)=d_{k}, k=1, \ldots, n$, and

$$
\begin{aligned}
\left(g \circ f_{*}^{1}\right)\left(c_{k}\right) & =g\left(c_{k} r_{*}\left(c_{k}\right)^{-1}\right) \\
& =c_{k} r_{*}\left(c_{k}\right) g\left(r_{*}\left(c_{k}^{-1}\right)\right) \\
& =c_{k} r_{*}\left(c_{k}\right) r_{*}\left(c_{k}^{-1}\right) \\
& =c_{k}, k=1, \ldots, m .
\end{aligned}
$$

This proves that $g^{-1}=f_{*}^{1}$, which finishes the proof.

Corollary 1.12. Let $M$ and $N$ be $C W$-complexes with unique 0 -cells, which we take to be their base points $*$ and $*^{\prime}$. Suppose that $M$ and $N$ are homotopic as spaces. There exists $m, n \in \mathbb{N}_{0}$ such that

$$
\left(M, M^{1}, *\right) \vee\left(D^{2}, S^{1}, *\right)^{\vee n} \cong\left(N, N^{1}, *^{\prime}\right) \vee\left(D^{2}, S^{1}, *^{\prime}\right)^{\vee m}
$$


thus, in particular,

$$
\Pi_{2}\left(M, M^{1}, *\right) \vee \Pi_{2}\left(D^{2}, S^{1}, *\right)^{\vee n} \cong \Pi_{2}\left(N, N^{1}, *^{\prime}\right) \vee \Pi_{2}\left(D^{2}, S^{1}, *^{\prime}\right)^{\vee m},
$$

where the free product is taken in the category of crossed modules; see Example 1.3.

The second part of this result was suggested by a referee of a previous version of this article.

Proof. The pointed spaces $(M, *)$ and $\left(N, *^{\prime}\right)$ are based homotopic. Let $P$ be the reduced mapping cylinder of some pointed homotopy equivalence $(M, *) \rightarrow\left(N, *^{\prime}\right)$, chosen to be cellular. Therefore both $M$ and $N$ are cellularly included in $P$ (provided with its usual CW-decomposition), and they intersect along their base points, both coinciding with the unique 0 -cell of $P$. The first result follows from the previous theorem and the fact that the inclusions of $M$ and $N$ in $P$ are homotopy equivalences. The second one follows directly from the 2-Dimensional van Kampen Theorem; see [5, 17, 10].

Note that we necessarily have $b_{1}\left(M^{1}\right)+n=b_{1}\left(N^{1}\right)+m=b_{1}\left(P^{1}\right)$. We will need to use this fact later. Here, if $K$ is a CW-complex, then $b_{1}(K)$ denotes its first Betti number. In addition $P$ is the reduced mapping cylinder of some pointed homotopy equivalence $(M, *) \rightarrow\left(N, *^{\prime}\right)$, chosen to be cellular.

It is easy to see that $\Pi_{2}\left(D^{2}, S^{1}\right)=(\mathbb{Z}, \mathbb{Z}, \mathrm{id}, \triangleright)$, where $\triangleright$ is the trivial action. We can prove this from the long homotopy exact sequence of $\left(D^{2}, S^{1}\right)$, or alternatively by using Whitehead's Theorem together with the explicit description of free crossed modules in Section 1.1.1.

The results of this subsection extend in a natural way for the case of the fundamental crossed complex of a CW-complex; see [18].

\section{Complements of KNotted Surfaces}

For the case of complements of knotted surfaces in $S^{4}$, it is convenient to work with handle decompositions, more flexible than CW-decompositions, considered in the previous section.

Let $M$ be a manifold with a handle decomposition, and let $M^{(n)}$ be the handlebody made out of the handles of $M$ of index smaller than or equal to $n$ (the $n$-handlebody of $M$ ). It is well known that a handle decomposition of $M$ determines a topological space $\hat{M}$ of the same homotopy type of $M$, with a $\mathrm{CW}$-decomposition where each $n$-handle of the manifold $M$ generates an $n$-cell of the CW-complex $\hat{M}$; see [42, Chapter 6] or [36. Chapter III], for example. Intuitively, $\hat{M}$ is obtained from $M$ by shrinking any $n$-handle to an $n$-cell going along its core. More precisely, it is possible to prove that, if $M$ is a manifold with a handle decomposition, then there exists a homotopy equivalence $M \rightarrow \hat{M}$ preserving the filtrations of $M$ and $\hat{M}$ given by their handle and cell decompositions, respectively, and such that the restriction maps $M^{(n)} \rightarrow \hat{M}^{n}$ are homotopy equivalences for each $n$; see [36, Proposition 3.4]. Given that all inclusion maps are cofibrations, it thus follows that there exists a filtered homotopy equivalence $M \rightarrow \hat{M}$.

Again let $M$ be a manifold with a handle decomposition. Suppose that the base point of $M$ is contained in one of the 0-handles of $M$. The above equivalence provides a homotopy equivalence $\left(M, M^{(1)}, *\right) \cong\left(\hat{M}, \hat{M}^{1}, *\right)$. Here the base point of $\hat{M}$ is taken to be one of the 0-cells of $\hat{M}$. Therefore, the results of the previous chapter apply with the obvious adaptations to the crossed module $\Pi_{2}\left(M, M^{(1)}\right)$. 

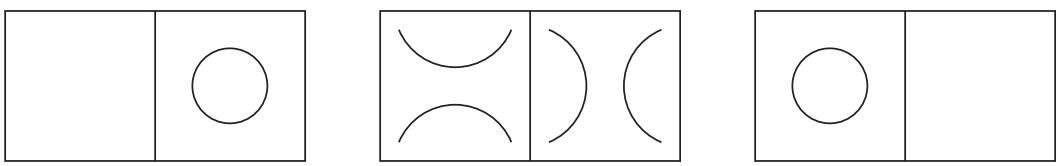

Figure 2. Morse Modifications, respectively a "Minimal Point", a "Saddle Point" and a "Maximal Point". Each of these modifications goes from left to right.
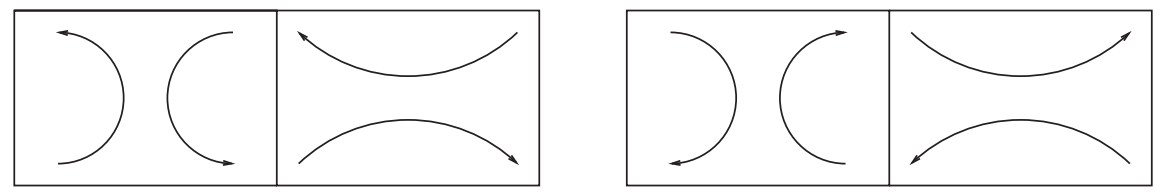

Figure 3. Oriented saddle point transitions.

2.1. Handle decompositions of complements of knotted surfaces. For details on knotted surfaces, in particular the movie presentation of them, we refer the reader to [14, 13, 12, 21]. We work in the smooth category.

2.1.1. Movies of knotted surfaces. Let $\Sigma \subset S^{4}=D_{-}^{4} \cup\left(S^{3} \times I\right) \cup D_{+}^{4}$ (where from now on $I=[-2,2])$ be a knotted surface. In other words $\Sigma$ is a (locally flat) embedding of a closed 2-manifold into $S^{4}$. We want to calculate $\Pi_{2}\left(M, M^{(1)}\right)$, where $M=S^{4} \backslash \nu(\Sigma)$. Here $\nu(\Sigma)$ is an (open) regular neighborhood of $\Sigma$ in $S^{4}$ and $M^{(1)}$ is the handlebody made out of the 0- and 1-handles of a handle decomposition of $M$ (the 1-handlebody of $M$ ). This will provide a description of the algebraic 2type of the complement of $\nu(\Sigma)$, in the form of a crossed module.

We need to construct a handle decomposition of the complement of $\nu(\Sigma)$ in $S^{4}$. Such a handle decomposition can be defined from a movie of $\Sigma$; see 22, 23, 12.

Up to isotopy, we can suppose that $\Sigma \subset S^{3} \times I$. Suppose that the projection on $I$ defines a Morse function on the knotted surface $\Sigma$. In particular, for each non-critical $t \in I$, the set $\Sigma_{t}=\Sigma \cap\left(S^{3} \times\{t\}\right)$ is a link in $S^{3}$ (a still of $\Sigma$ ). Between critical values, the link $\Sigma_{t}$ will undergo an isotopy of $S^{3}$. At critical points of index 0,1 or 2 , the link $\Sigma_{t}$ will go through Morse modifications, called, respectively, "Minimal Points", "Saddle Points" and "Maximal Points"; see Figure 2, The 1parameter family of links $t \mapsto \Sigma_{t}$, with the modifications at non-generic points, will define what is called a "movie" of the knotted surface $\Sigma \subset S^{4}$.

If the knotted surface $\Sigma$ is oriented, then each link $\Sigma_{t}$ appearing in the movie (now called an oriented movie) of $\Sigma$ will have a natural orientation. See Figure 3 for the oriented version of the saddle point moves. Any oriented movie defines an oriented knotted surface, up to isotopy.

2.1.2. Hyperbolic splittings and knots with bands. Let $\Sigma \subset S^{4}=D_{-}^{4} \cup\left(S^{3} \times I\right) \cup D_{+}^{4}$ be a knotted surface presented by a movie $t \mapsto \Sigma_{t}$. Recall that $I=[-2,2]$. By using isotopy, we can suppose that $\Sigma \subset S^{3} \times[-1,1]$, and, moreover, that all minimal points occur in $S^{3} \times\{-1\}$, all maximal points occur in $S^{3} \times\{1\}$, and all saddle points occur in $S^{3} \times\{0\}$; see for example [12, Chapter 1] or [28]. This is a well know result. This type of movie of knotted surfaces is usually called a "hyperbolic splitting". 


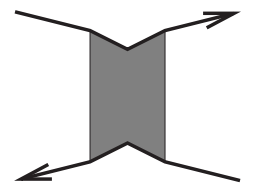

Figure 4. A bit of a knot with bands.

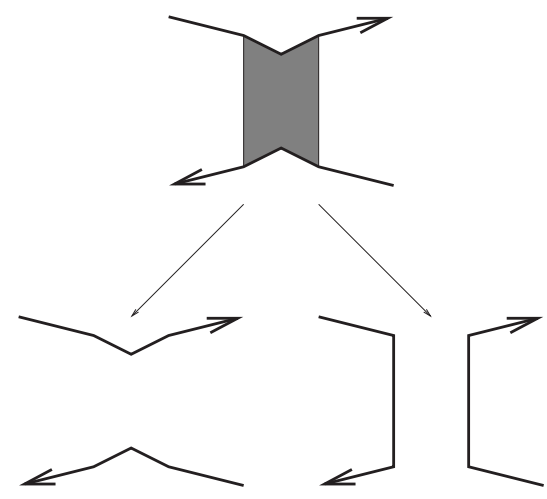

Figure 5. A piece of a knot with bands together with its postknot and pre-knot (from left to right).

Consider a knotted surface $\Sigma$ represented by a hyperbolic splitting. Therefore, for all $t$ in $(-1,0)$, the still $\Sigma_{t}=\Sigma \cap\left(S^{3} \times\{t\}\right)$ of $\Sigma$ will be an unlink with a fixed number of components, and $\Sigma_{t}$ will undergo an isotopy of $S^{3}$ in this interval. The same is true for $t \in(0,1)$. At $t=0$ the link $\Sigma_{t}$ will undergo saddle point transitions; see Figure 3. We have in addition minimal and maximal points at $t=-1$ and $t=1$, respectively.

All this information used for constructing a knotted surface is highly redundant, 31. In fact, the knotted surface constructed in this way depends only on the saddle point transitions at $t=0$, as well as the configuration immediately before and after $t=0$; see for example 28] for a proof.

The saddle point transitions which happen at $t=0$ can be encoded by a knot with bands; see 28, 43, 12. Another usual (and equivalent) presentation is to use marked vertex diagrams; see [31, 12, 49]. The former are more useful for our purposes since with them we can suppose that the configuration immediately after the saddle points is a standard diagram of the unlink.

A knot with bands is a knot together with some $I \times I$ bits, intersecting the knot along $\partial I \times I$. A knot with bands is said to be oriented if we have orientations on the thin edges of it, having the configuration of Figure 4, or its mirror image, at the edges incident to a band. Henceforth, all knots with bands will be oriented. A knot with bands $K$ determines two oriented knots $K_{+}$and $K_{-}$called the post-knot and pre-knot of $K$; see Figure 5. Note that our convention is opposite of the one in [43].

Let $K$ be a knot with bands such that the post- and pre-knots of $K$ are oriented unlinks. We can construct an oriented knotted surface by choosing an isotopy from the post-knot $K_{+}$of $K$ to the standard unlink diagram for $t>0$, and by capping 
all the circles of it in the obvious way, and analogously for the interval $[-1,0)$. The final result does not depend on the choices made, up to isotopy. In fact, it depends only on the isotopy class of $K$.

In this article we will consider this description of knotted surfaces. However, we will need to use the movie picture associated to a presentation of a knotted surface by knot with bands when constructing the handle decomposition of its complement.

2.1.3. Construction of the handle decomposition. Let $\Sigma \subset S^{4}=D_{-}^{4} \cup\left(S^{3} \times I\right) \cup D_{+}^{4}$ be a knotted surface presented by a movie $t \mapsto \Sigma_{t}$. Suppose that $\Sigma \subset S^{3} \times[-1,1]$. Therefore $\Sigma$ is provided with a Morse function (the projection on $I$ ) and, away from critical points, $\Sigma_{t}$ is a link in $S^{3}$. There exists a natural handle decomposition of the complement $M$ of an open regular neighborhood $\nu(\Sigma)$ of $\Sigma$ (in principle defined up to handle-slides and isotopy) where minimal/maximal points will induce $1 / 3$-handles of the decomposition, and saddle points induce 2-handles; see [23, section 6.2], [12, 3.1.1], or [22. This is very easy to visualize in dimension 3 . To calculate the fundamental crossed module $\Pi_{2}\left(M, M^{(1)}\right)$, however, we need an explicit description of this handle decomposition. We now follow [12, 3.1.1] and [23. 6.2], where the missing bits of our description can be found.

Let $K$ be a knot with bands, representing the knotted surface $\Sigma \subset S^{3} \times[-1,1]$. Choose a regular projection of $K$. Let $t \mapsto \Sigma_{t}$ be an associated movie for $\Sigma$, a hyperbolic splitting. For simplicity (and without loss of generality), we will suppose that the post-knot of $K$ is a standard diagram of the unlink (a disjoint union of unknotted circles). This will fix a handle decomposition of the complement $M$ of a regular neighborhood of $\Sigma$, up to isotopy. From now on we will suppose that all knots with bands representing knotted surfaces are on this form.

For any set $A$ in $S^{4}=D_{-}^{4} \cup\left(S^{3} \times I\right) \cup D_{+}^{4}$, let $A_{t}=A \cap\left(S^{3} \times\{t\}\right)$ and $A_{\leq t}=A \cap\left(\left(S^{3} \times[-2, t]\right) \cup D_{-}^{4}\right)$. Then there exists some small $\epsilon>0$ such that the topology of $M_{\leq t}$ does not change in the intervals $[-2,-1-\epsilon],[-1+\epsilon,-\epsilon]$, $[\epsilon, 1-\epsilon]$ and $[1+\epsilon, 2]$. In between these intervals, the manifold $M_{\leq t}$ will undergo attachment of handles of index, respectively, $1,2,3$ and 4 .

The manifold $M_{\leq(-1+\epsilon)} \cong M_{\leq-\epsilon}$ is a 4-manifold obtained by attaching $n$ 1handles to $D^{4} \cong M_{\leq(-1-\epsilon)}$. Here $n$ is the number of components of the pre-knot of $K$. In fact a Kirby diagram for $M_{\leq-\epsilon}$ is obtained from the pre-knot $K_{-}$of $K$ by turning the circles of it (considered to be 0 -framed) into dotted circles, in the notation of Kirby, 29]; see [23, Section 6.2]. This is also clear from the construction in [12, 3.1.1].

The manifold $M_{\leq \epsilon}$ is obtained from $M_{\leq-\epsilon}$ by attaching 2-handles, each of which is determined by a band of $K$. For each $t \in[\epsilon, 1-\epsilon]$, the manifold $M_{\leq t}$ is therefore a 4-dimensional handlebody made from 0-, 1- and 2-handles, the 2-handlebody $M^{(2)}$ of the knotted surface complement $M$.

We can easily obtain a Kirby diagram for $M^{(2)}$ from the knot with bands $K$; cf. 23, Section 6.2]. Consider a dotted circle for each circle of $K_{-}$, the pre-knot of $K$. Then each 2-handle should be attached along a framed circle $S^{1} \times I$ encircling one of the bands of $K$, with framing parallel to the core of it; see Figure 6. In particular each 2-handle attaches along a 0 -framed circle. To understand the subsequent attachment of 3-handles, it is convenient to draw the framed circle $S^{1} \times I$ encircling each band of $K$ in a way such that the framing of it goes along almost the entire length of the band, as in Figure 6 . 

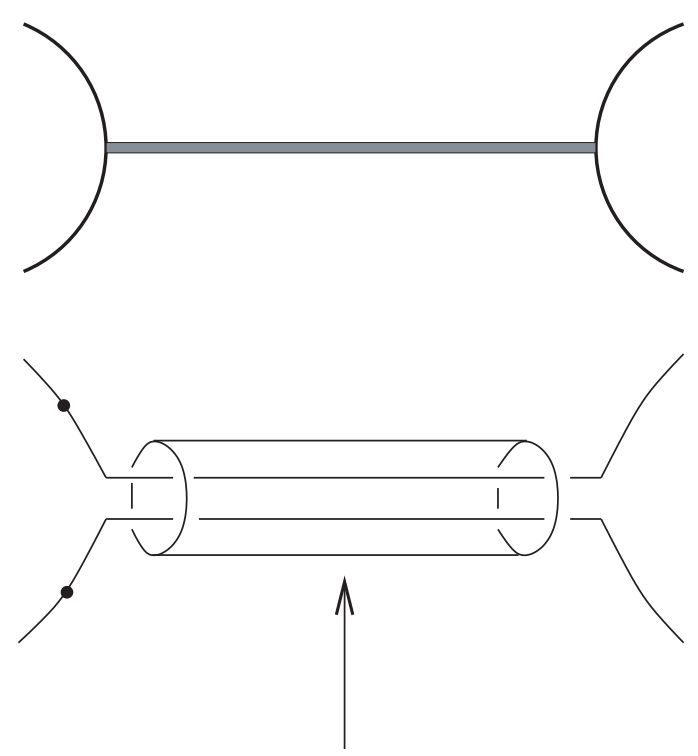

framed circle $S^{1} \times I$ along which a 2 -handle attaches

Figure 6. Portion of the Kirby diagram for the 2-handlebody $M^{(2)}$ of the complement of a knotted surface obtained from a knot with bands. The original diagram appears on top.

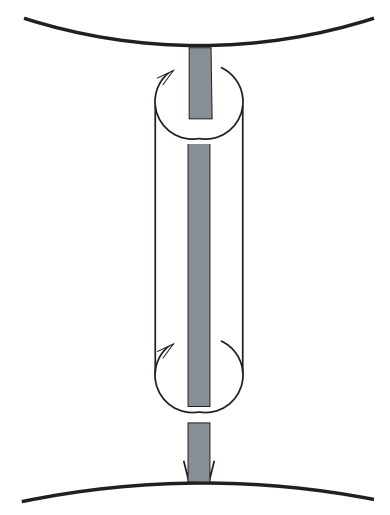

Figure 7 . The oriented framed circle $S^{1} \times I$ determined by a band of a knot with bands with its core oriented.

To describe the attaching map of each 2-handle (and not simply the attaching region) we need an orientation of its attaching sphere. Such orientation can be fixed by an orientation of the core of the associated band; see Figure 7 . In subsequent drawings of knots with bands, there will be arrows denoting the orientation of both the thin and fat strands of it; see Figure 8 .

The handles of index 3 attach along regions diffeomorphic with $S^{2} \times I$. In the case of complements of knotted surfaces, 3-handles correspond to maximal points. The attaching sphere of each 3 -handle will be a sphere $S^{2}$ containing one of the circles of the post-knot of $K$ in the region inside it, as in Figure 9, see [12, 3.1.1] 


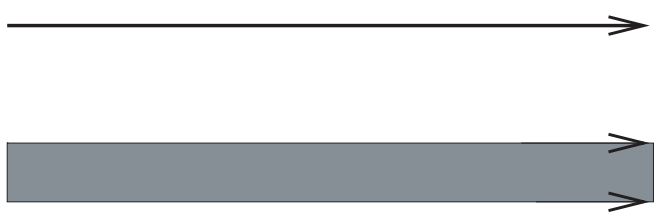

Figure 8. Denoting orientations of thin and fat components of a knot with bands.

and [23, 6.2]. Recall that we suppose that the post-knot $K_{+}$of $K$ is a standard diagram for the unlink.

In the Kirby diagram for the knotted surface complement $M$, the configuration can be more complicated due to the previous attachment of 1- and 2-handles. We can suppose that each of the attaching spheres $S^{2}$ determined by the circles of $K_{+}$ intersects the framed circles $S^{1} \times I$ determined by the bands of $K$ transversally, thus along a disjoint union of circles $S^{1}$, circles which we can also suppose go around the corresponding band of $K$. This type of intersection will be called essential. Therefore, in the vicinity of the circles of $K_{+}$, the configuration of the Kirby diagram of $M$ will look like Figure 10.

Note that since we perform surgery on the framed knots $S^{1} \times I$ appearing in Figure 10, the shown embedded sphere $S=S^{2}$ is well defined. In fact the intersection of $S$ with the previously attached 2-handles is, in this particular case, a disjoint union of three disks $U_{1}, U_{2}$ and $U_{3}$, whose boundary is the intersection of $S$ with the framed circles $S^{1} \times I$ determined by the bands; see Figure 10. These disks $U_{1}, U_{2}$ and $U_{3}$ are parallel to the core of the corresponding 2-handles of $M$. This remark continues to hold for more complicated configurations, with the obvious adaptations. Therefore we have:

Lemma 2.1. The attaching sphere of each 3-handle is a sphere $S^{2}$ containing one, and only one, of the circles of the post-knot $K_{+}$of $K$ in the region inside it, as in Figure 9. We can suppose that each attaching sphere $S^{2}$ intersects the framed circles $S^{1} \times I$ determining the attachment of 2-handles transversally, thus each connected component of the intersection is a circle $S^{1}$, which furthermore we can suppose is linking the associated band of $K$, locally (an essential intersection). Moreover, the attaching sphere $S^{2}$ of each 3-handle intersected with the 2-handles is a disjoint union of disks (one for each essential intersection), each of which is parallel to the core of the corresponding 2-handle. The boundary of each of these disks is the corresponding connected component of the intersection of the attaching sphere $S^{2}$ with the framed circles determining the attachment of 2-handles.

Note that the framed circle determined by a band may intersect the attaching sphere for a 3-handle more than once.

Finally we attach a 4 -handle at $t=2$. By the Cellular Approximation Theorem, this 4-handle will not affect the calculation of $\Pi_{2}\left(M, M^{(1)}\right)=\Pi_{2}\left(M^{(3)}, M^{(1)}\right)$.

We have thus defined a handle decomposition of the complement $M$ of a knotted surface $\Sigma$ if we are given a knot with bands $K$ representing it, chosen so that the the post-knot of $K$ is a standard diagram of the unlink.

Summarizing, a Kirby diagram for $M$ will have a dotted circle for each circle of the pre-knot $K_{-}$of $K$, considered to be 0 -framed. Then, each band of $K$ will induce a 2-handle of the complement, and the attaching region of it is determined 


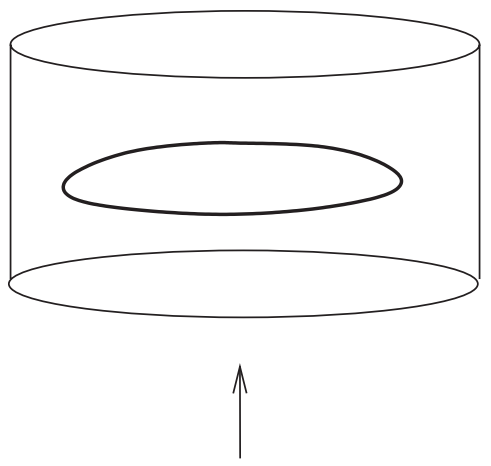

attaching sphere $S^{2}$ for a 3-handle

FiguRE 9. The attaching sphere for a 3-handle determined by a circle of the post-knot of $K$.

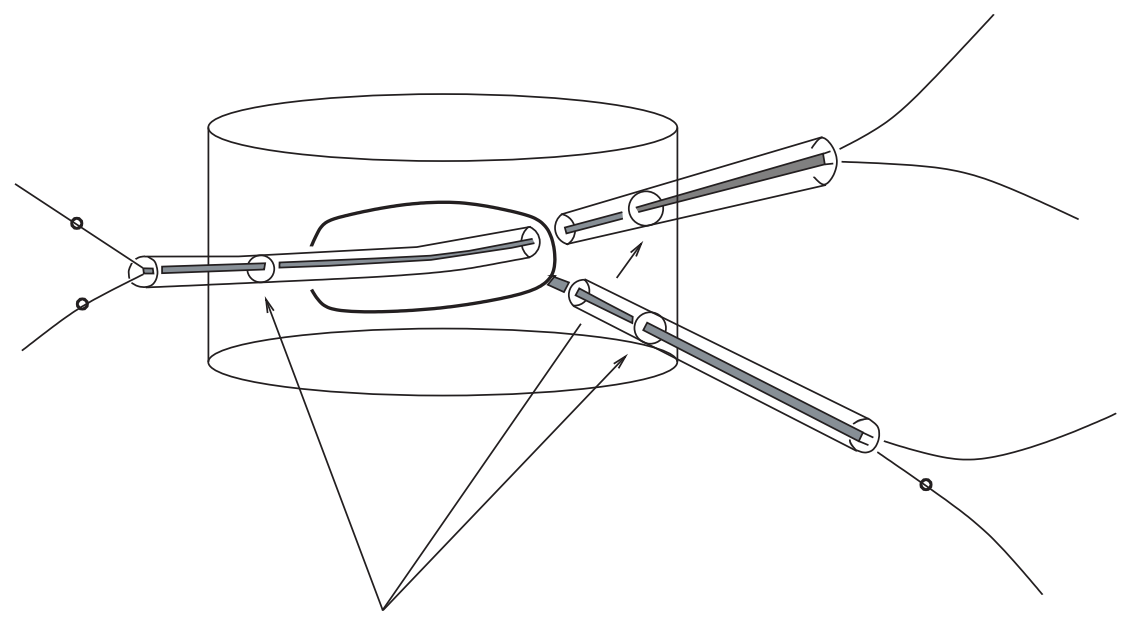

Intersections of the attaching sphere $S^{2}$ with the framed knot determined by the bands

Figure 10. Attaching sphere for a 3-handle in the Kirby diagram determined by a knot with bands representing a knotted surface. Note that the bands appearing in the figure do not belong to the Kirby diagram, whose set of dotted circles will be the pre-knot of the knot with bands showing.

by a framed circle $S^{1} \times I$, encircling the band, with framing parallel to the core of it (thus yielding a 0-framed circle), and going along almost the entire length of the band; see Figure 7. Then we attach a 3-handle for each circle of the post-knot of $K$, which we suppose to be a standard diagram of the unlink. The attaching sphere $S^{2}$ of each 3-handle contains one, and only one, of the components of $K_{+}$ in the region inside it, and it intersects the framed circles determined by the bands of $K$ transversally, so that each connected component of the intersection (a circle $\left.S^{1}\right)$ goes around the corresponding band of $K$. 


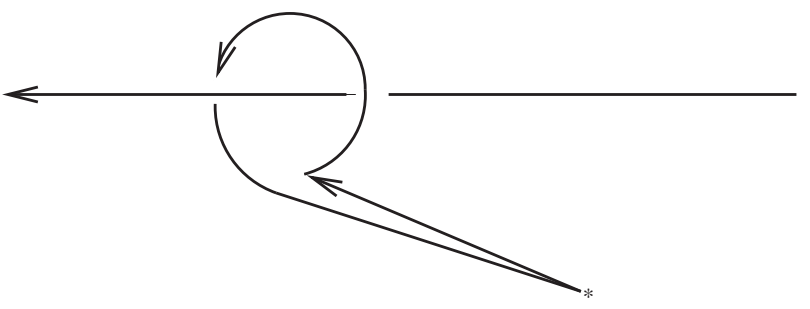

Figure 11. Defining an element of the fundamental group of a knot complement.

Even though it is not strictly necessary to retain the bands of $K$ to understand the Kirby diagram, it is useful to consider them for determining the fundamental crossed module of the complement.

2.2. The calculus. Let $\Sigma \subset S^{4}=D_{-}^{4} \cup\left(S^{3} \times I\right) \cup D_{+}^{4}$ be a knotted surface defined by a knot with bands $K$; thus $\Sigma \subset S^{3} \times[-1,1]$. Here $I=[-2,2]$. We suppose that the surface $\Sigma$, thus $K$, is oriented. Choose a regular projection $p$ of $\mathbb{R}^{3}$ onto a hyperplane of it such that $p$ is a regular projection of $K$, thus defining a natural base point $*$ of $S^{3}$, the "eye of the observer"; see 14. Consider the handle decomposition of the knotted surface complement $M=S^{4} \backslash \nu(\Sigma)$ just described, and let $M^{(1)}, M^{(2)}$ and $M^{(3)}$ be the 1-, 2- and 3-handlebodies of it. As usual, if $A \subset S^{4}$ is a set, we denote $A_{\leq t}=A \cap\left(\left(S^{3} \times[-2, t]\right) \cup D_{-}^{4}\right)$. Recall that $\nu(\Sigma)$ denotes an open regular neighbourhood of $\Sigma$ in $S^{4}$.

2.2.1. Wirtinger relations. The fundamental group of $M_{\leq-\epsilon} \cong M^{(1)}$, a free group, is isomorphic with the fundamental group of the complement of the pre-knot $K_{-}$of $K$ in $S^{3}$, the free group on the components of $K_{-}$, since $K_{-}$is an unlinked union of unknotted circles. We can define a presentation of $\pi_{1}\left(S^{3} \backslash K_{-}\right)$by considering the Wirtinger Presentation; see for example [11. Therefore each arc (upper crossing) of the projection $p\left(K_{-}\right)$of $K_{-}$gives a generator of $\pi_{1}\left(S^{3} \backslash K_{-}\right)$, and each crossing yields a relation; see Figures 11 and 12. For such a presentation, the base point will stay at the "eye of the observer" of the chosen projection. Notice that we need to consider orientations on the knot diagram $p\left(K_{-}\right)$so that these elements are well defined. Therefore, it is at this point that we need to introduce the (probably artificial) restriction that all the knotted surfaces that we consider are oriented, so that we can orient the associated knot with bands $K$, providing compatible orientation of $K_{-}$and $K_{+}$. The final result will certainly not depend on the chosen orientation of $\Sigma$.

2.2.2. Saddle point relations. When we pass the saddle points at $t=0$, we attach 2-handles. Therefore, by Whitehead's Theorem (Theorem 1.8), for $t \in[\epsilon, 1-\epsilon]$, the crossed module $\Pi_{2}\left(M_{\leq t}, M_{\leq t}^{(1)}\right)=\Pi_{2}\left(M^{(2)}, M^{(1)}\right)$ is the free crossed module on the set of 2-handles and their attaching maps, over the group $\pi_{1}\left(M^{(1)}\right) \cong \pi_{1}\left(S^{3} \backslash K_{-}\right)$, the free group on the set of circles of the pre-knot $K_{-}$of $K$.

Each band of $K$ will define a 2-handle of the knotted surface complement $M$. However, some details are needed in order to specify the element of $\pi_{2}\left(M^{(2)}, M^{(1)}\right)$ defined by it as well as its boundary in $\pi_{1}\left(M^{1}\right)$. These are only defined up to acting and conjugation by a certain element of $\pi_{1}\left(M^{(1)}\right)$; see Section 1.2.1. 

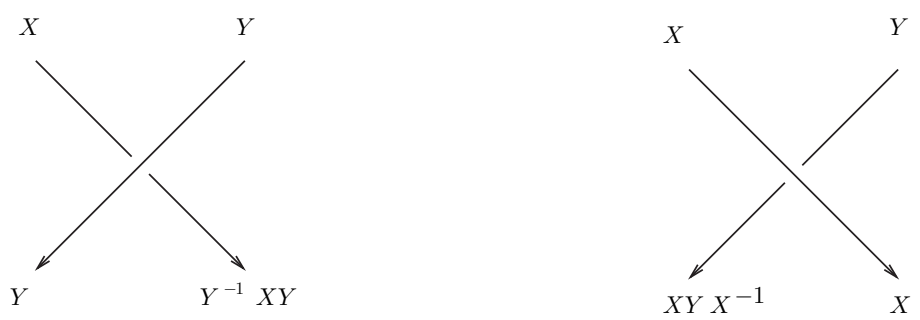

FiguRE 12. Wirtinger Relations.

To make our discussion clearer, suppose that the knot with bands $K$ is such that each band of $K$ always has the same side facing upwards, for the chosen regular projection of $K$. Standard arguments prove that any knot with bands can be isotoped so that it is in this form, whilst keeping the post-knot of it as being a standard diagram for the unlink. From now on any regular projection of a knot with bands will be supposed to have this special form.

To specify the element of $\pi_{2}\left(M^{(2)}, M^{(1)}\right)$ induced by each band, we will need to consider an orientation of the core of it (therefore defining the attaching map of the associated 2-handle up to isotopy), as well as an arc (upper crossing) of the band in the chosen regular projection $p(K)$ of $K$. This is similar to the definition of the Wirtinger presentation of knot complements.

The exact definition of these elements of $\pi_{2}\left(M^{(2)}, M^{(1)}\right)$ is the following; cf. Figure 7 Choose a base point $*^{\prime}$ on the upper part (with respect to the projection $p$ ) of the framed circle $S^{1} \times I$ determined by the band. Consider a based circle $\left(c, *^{\prime}\right)$ contained in the framed circle, so that $c$ goes around the band. The circle $\left(c, *^{\prime}\right)$ is the boundary of a certain based disk $\left(U, *^{\prime}\right)$ embedded in the 2-handle associated with the band, parallel to its core, by definition of attachment of 2handles. Therefore the based disk $\left(U, *^{\prime}\right)$ defines an element of $\pi_{2}\left(M^{(2)}, M^{(1)}, *^{\prime}\right)$ as in Section 1.2.1 and its boundary in $\pi_{1}\left(M^{(1)}, *^{\prime}\right)$ is exactly $c$. Note that the attaching map of the attached 2-handle is well defined since the core of the band is oriented.

Suppose that $*^{\prime}$ can be connected to the base point * (the "eye of the observer" of $p(K)$ ) by a straight line which does not intersect the knot with bands. The natural isomorphism $\Pi_{2}\left(M^{(2)}, M^{(1)}, *^{\prime}\right) \rightarrow \Pi_{2}\left(M^{(2)}, M^{(1)}, *\right)$ defined by this curve determines the element of $\pi_{2}\left(M^{(2)}, M^{(1)}, *\right)$ specified by an arc of a band in $p(K)$. (It is easy to see that this element depends only on the arc of a band to which the base point $*^{\prime}$ belongs.)

To determine the boundary in $\pi_{1}\left(M^{(1)}\right)$ of the elements of $\pi_{2}\left(M^{(2)}, M^{(1)}\right)$ defined by arcs of bands, we can use the following proposition.

Proposition 2.2. The boundary $\partial(e) \in \pi_{1}\left(M^{(1)}\right) \cong \pi_{1}\left(S^{3} \backslash K_{-}\right)$of the element $e \in \pi_{2}\left(M^{(2)}, M^{(1)}\right)$, determined by an arc of a band, satisfies the relations of Figure 13. Note that $\pi_{1}\left(M^{(1)}\right)$ is isomorphic to the fundamental group of the complement of the pre-knot $K_{-}$of $K$, itself presented by the Wirtinger presentation of knot complements.

Proof. This follows immediately from the definition of the Wirtinger presentation, as well as the definition of the elements determined by an arc of a band. We refer to Figure 14 for the proof in one particular case (the second case of Figure 13). 

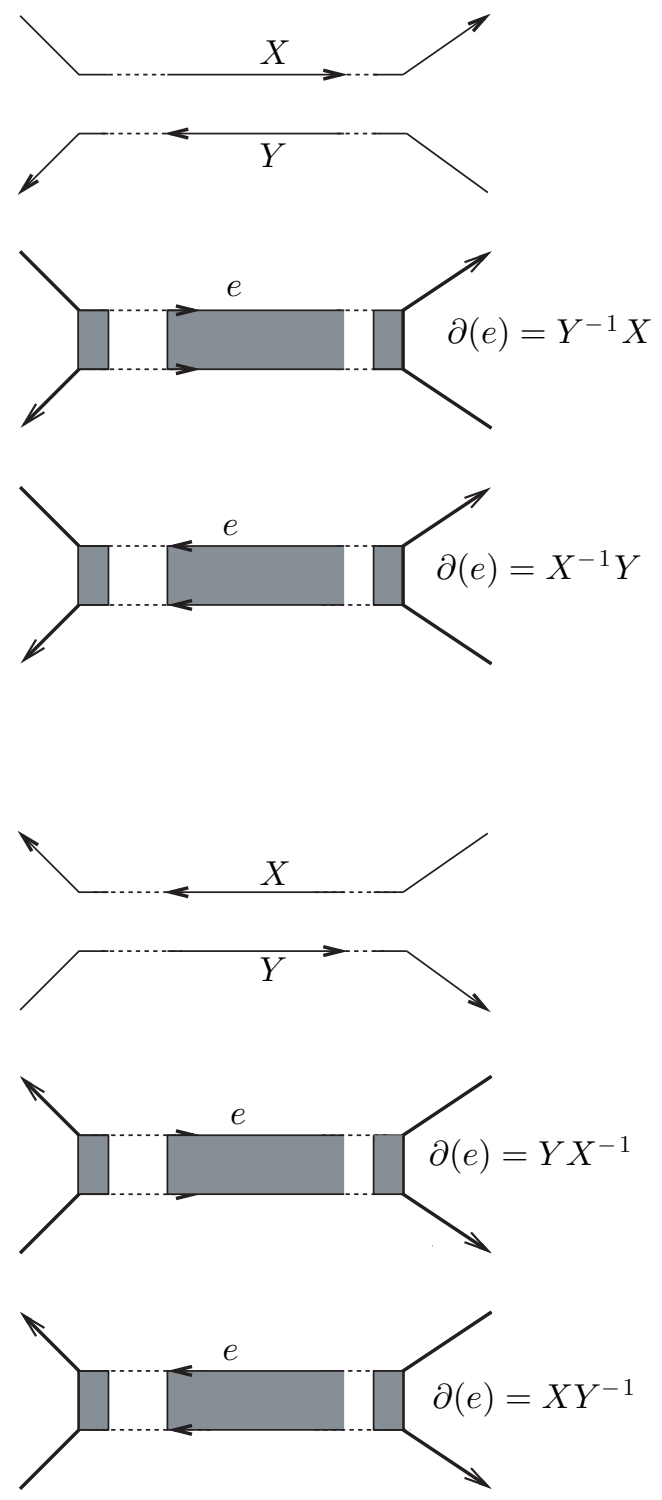

Figure 13. The boundary $\partial(e) \in \pi_{1}\left(M^{(1)}\right)$ of the element $e \in$ $\pi_{2}\left(M^{(2)}, M^{(1)}\right)$ determined by an arc of a band of a knot with bands. Note that $\pi_{1}\left(M^{(1)}\right) \cong \pi_{1}\left(S^{3} \backslash K_{-}\right)$is represented by the Wirtinger presentation of knot complements. The knots without bands appearing in the figure are the pre-knots of the remaining knots with bands.

The element of $\pi_{2}\left(M^{(2)}, M^{(1)}\right)$ determined by a band of a knot with bands depends on the arc chosen. The exact dependence on the arc is described in the following proposition. 

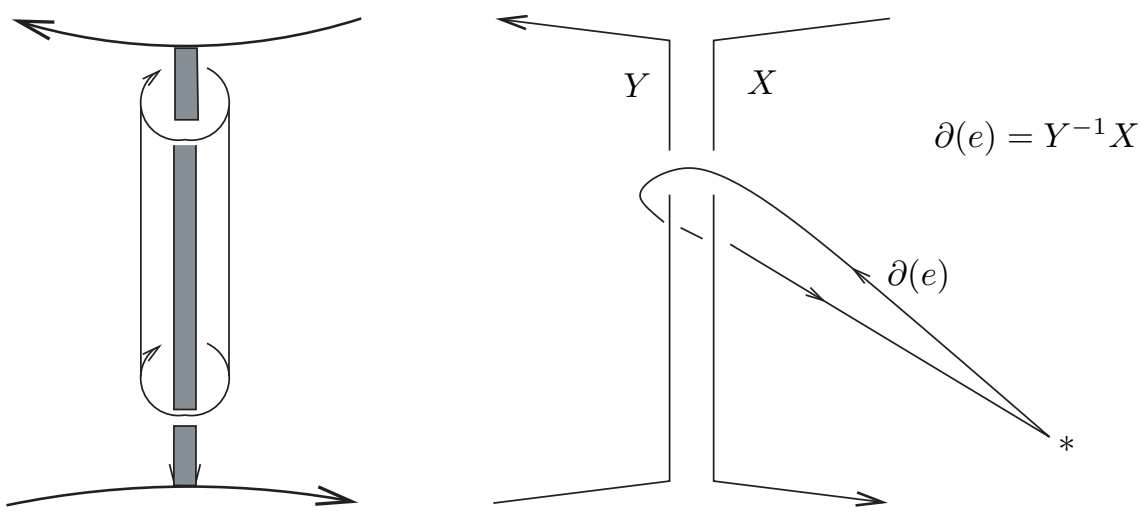

Figure 14. Calculation of the boundary in $\pi_{1}\left(M^{(1)}\right)$ of the element $e$ of $\pi_{2}\left(M^{(2)}, M^{(1)}\right)$ determined by an arc of a band of a knot with bands.
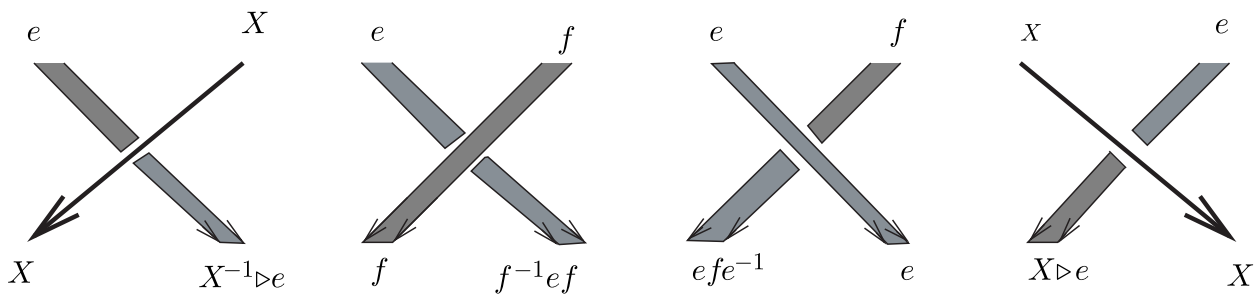

Figure 15. Relations satisfied by the elements of $\pi_{2}\left(M^{(2)}, M^{(1)}\right)$ specified by an arc of a band of a knot with bands.

Proposition 2.3. The elements of $\pi_{2}\left(M^{(2)}, M^{(1)}\right)$ determined by arcs of bands of a knot with bands satisfy the relations of Figure 15.

Proof. This follows essentially from the fact that if we use two different paths $\gamma_{1}$ and $\gamma_{2}$ from the base point of a CW-space $N$ to the base point of a 2-cell $c$ to identify the 2-cell $c$ with an element of $\pi_{2}\left(N^{2}, N^{1}, *\right)$, then the corresponding elements $c_{\gamma_{1}}$ and $c_{\gamma_{2}}$ of $\pi_{2}\left(N^{2}, N^{1}, *\right)$ are related by $c_{\gamma_{1}}=\left(\gamma_{1} \gamma_{2}^{-1}\right) \triangleright c_{\gamma_{2}}$. To prove the middle bits, we also need to use the second condition of the definition of crossed modules, together with the relations of Figure 13

We have:

Proposition 2.4. Let $\Sigma$ be an oriented knotted surface, and let $K$ be an oriented knot with bands representing $\Sigma$, provided with some regular projection. As usual, suppose that the post-knot $K_{+}$of $K$ is a standard diagram for the unlink, and that each band of $K$ always has the same side facing upwards. Let $M=S^{4} \backslash \nu(\Sigma)$ be the complement of an open regular neighborhood of $\Sigma$, with handle decomposition as above. For each band of $K$ choose an arc of it, therefore defining an element of $\pi_{2}\left(M^{(2)}, M^{(1)}\right)$, as well as its boundary in $\pi_{1}\left(M^{(1)}\right)$, the free group on the set of circles of the pre-knot $K_{-}$of $K$ (itself presented by the Wirtinger presentation of knot complements); see Figure 13. Then the crossed module $\Pi_{2}\left(M^{(2)}, M^{(1)}\right)$ is the free crossed module on this map from the set of bands of $K$ into $\pi_{1}\left(M^{(1)}\right) \cong \pi_{1}\left(S^{3} \backslash K_{-}\right)$. 


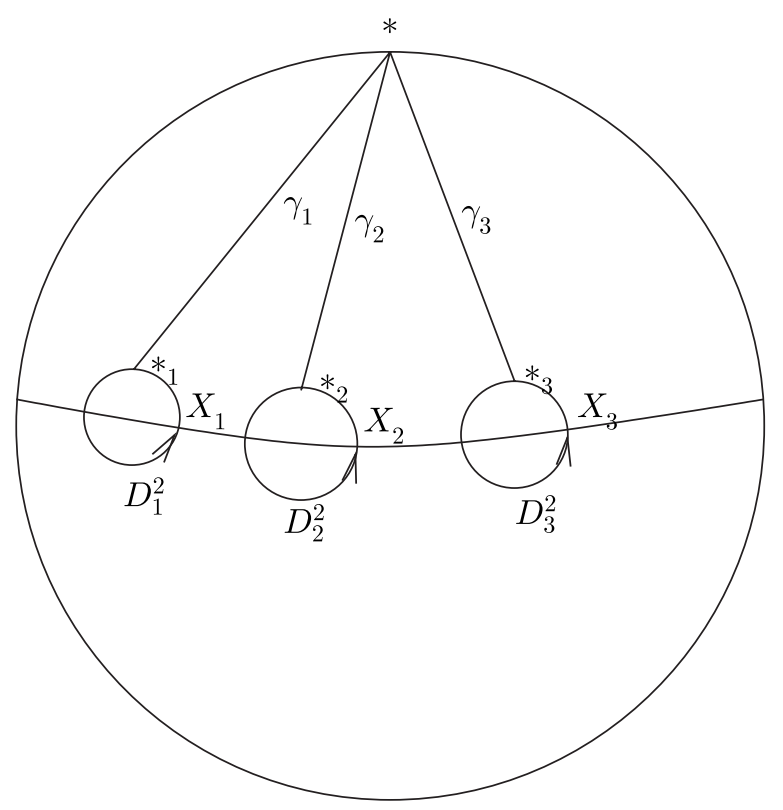

Figure 16. Figure relative to Lemma 2.5.

2.2.3. The 2-relations at maximal points. Given the drawing of the attaching sphere of a 3-handle in Figure 10, it is possible to determine the 2-relations (implied by Theorem 1.10) coming from the attachment of a 3-handle at a maximal point. First of all we need to determine the element $r \in \pi_{2}\left(M^{(2)}, M^{(1)}\right)$ induced by the attaching map of each 3-handle; see Theorem 1.10. We will need the following intuitive lemma, whose straightforward proof is left to the reader.

Consider the 2-sphere $S^{2}$ having the north pole as a base point $*$. Remove 2disks $D_{i}^{2}$, where $i=1,2, \ldots, n$ from $S^{2}$, whose middle points lie in the equator of $S^{2}$, with base points $*_{i}$ at their highest points; see Figure 16 for the case $n=3$. Consider the obvious paths $\gamma_{i}$ from $*_{i}$ to $*$ along a meridian. Then $S^{2}$ is obtained from $S^{2} \backslash\left(\bigcup_{i=1}^{n} D_{i}^{2}\right)$ by attaching a 2 -cell for each $i=1, \ldots, n$ along the obvious attaching map $X_{i}$ shown in Figure 16. The paths $*_{i} \rightarrow *$ permit us to associate elements $e_{i}$ of $\pi_{2}\left(S^{2}, S^{2} \backslash\left(\bigcup_{i=1}^{n} D_{i}^{2}\right)\right)$ to each disk $D_{i}^{2}$.

Lemma 2.5. The product $e_{1} e_{2} \ldots e_{n} \in \pi_{2}\left(S^{2}, S^{2} \backslash\left(\bigcup_{i=1}^{n} D_{i}^{2}\right)\right)$ coincides with the element of $\pi_{2}\left(S^{2}, S^{2} \backslash\left(\bigcup_{i=1}^{n} D_{i}^{2}\right)\right)$ naturally defined by the oriented $S^{2}$.

Let $K$ be an oriented knot with bands so that the post- and pre-knots $K_{+}$and $K_{-}$ of it are oriented unlinks, with the post-knot of $K$ being a standard diagram of the unlink. Suppose also that each band of $K$ always has the same side facing upwards. Consider the natural handle decomposition of the complement $M$ of the oriented knotted surface $\Sigma$ determined by $K$. Let $c_{1}, \ldots, c_{m}$ be the circles of the post-knot of $K$, thus there exists one 3 -handle of $M$ for each $c_{i}$. For each $i=1, \ldots, m$, let $S_{i}^{2}$ be a sphere (the attaching sphere of the associated 3-handle) containing the circle $c_{i}$ in the region inside it, so that the other circles $c_{j}$ are outside. As before, suppose that $S_{i}^{2}$ intersects the framed circles determined by the bands of $K$, transversally, along the equator, so that each connected component of the intersection is a circle 
$S^{1}$ encircling the corresponding band; see Lemma 2.1. Recall that this type of intersections are called essential.

Each sphere $S_{i}^{2}$, where $i=1,2, \ldots, m$, has a natural orientation induced by the orientation of a ball in $S^{3}$. Let $\left\{e_{i}^{1}, \ldots, e_{i}^{n_{i}}\right\}$ be the set of essential intersections of the framed circles determined by the bands of $K$ with $S_{i}^{2}$ (note that each framed circle may intersect $S_{i}^{2}$ more than once), ordered as in Figure 16. By "intersections" we mean connected components of the intersection of $S_{i}^{2}$ with the framed circles determined by the bands.

The core of each band is oriented, by assumption. Let $\theta_{i}^{j}=1$ if, with respect to the attaching sphere $S_{i}^{2}$, the bit of band determining $e_{i}^{j}$ is pointing outwards, and -1 otherwise.

Each intersection $e_{i}^{j}$ induces an element $e_{i}^{j} \in \pi_{2}\left(M^{(2)}, M^{(1)}\right)$, determined by the arc of band to which $e_{i}^{j}$ is encircling. This group element is the one provided from the fact that the intersection $e_{i}^{j}$ bounds a disk embedded in the corresponding 2handle, parallel to its core. This disk is a connected component of the intersection of the attaching sphere $S_{i}^{2}$ with the 2-handle; see Lemma 2.1. This discussion implies:

Theorem 2.6. The crossed module $\Pi_{2}\left(M, M^{(1)}\right)$ can be presented by the map from the set of bands of $K$ into $\pi_{1}\left(S^{3} \backslash K_{-}\right)$defined in Proposition 2.4, considering the 2 -relation

$$
\prod_{j=1}^{n_{i}}\left(e_{i}^{j}\right)^{\theta_{i}^{j}}=1
$$

well defined up to cyclic permutations, for each circle $c_{i}$ of the post-knot of $K$.

Proof. The crossed module $\Pi_{2}\left(M^{(2)}, M^{(1)}\right)$ is the free crossed module on the set of bands of $K$ and the attaching maps of the associated 2-handles; see Proposition 2.4. The manifold $M^{(3)}$ obtained by attaching the 3-handles determined by the circles of $K_{+}$to $M^{(2)}$ is homotopic to the space obtained by attaching a 3-cell along each circle $S_{i}^{2}$.

We now need to apply Theorem 1.10. The set of 2-relations yielding the fundamental crossed module $\Pi_{2}\left(M^{(3)}, M^{(1)}\right)$ is given by the elements $r_{i}$ of $\pi_{2}\left(M^{(2)}, M^{(1)}\right)$ determined by the attaching sphere $S_{i}^{2}$ of each 3-handle; here $i=1, \ldots, m$, where $m$ is the number of circles of the post-knot $K_{+}$of $K$. In terms of the generators of $\pi_{2}\left(M^{(2)}, M^{(1)}\right)$ determined by the bands of the knot with bands, each of these elements is exactly given by the formula $r_{i}=\prod_{j=1}^{n_{i}}\left(e_{i}^{j}\right)^{\theta_{i}^{j}}$. This follows from lemmas 2.5 and 2.1

Note that the 2-relation motivated by the attachment of a 3 -handle at a maximal point in principle depends on the chosen sphere $S^{2}$ containing the corresponding circle of the post-knot $K_{+}$of $K$ in the region inside it. The configuration of this attaching sphere will usually be as in Figure 10, so some bands of $K$ may be entirely in the region inside the chosen attaching sphere. In particular they do not appear in the 2-relation motivated by the attachment of the 3 -handle. This is because the intersection of the attaching sphere with the framed circle determined by the band is empty; see Figure 17 for one such example.

2.2.4. Simple examples. Consider the surface $\Sigma_{1}$ represented by the knot with bands $K_{1}$ of Figure 18. Therefore $\Sigma_{1}$ is a trivial embedding of a sphere $S^{2}$ in $S^{4}$. Let $M_{1}$ be the complement of an open regular neighborhood of $\Sigma_{1}$, provided 


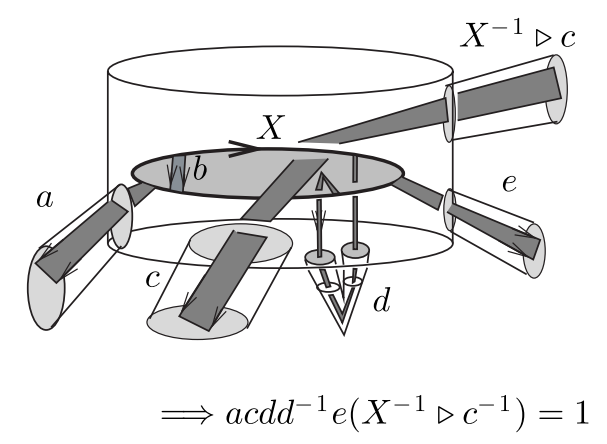

Figure 17. The 2-relations implied by the attachment of a 3handle at a maximal point in one particular example.
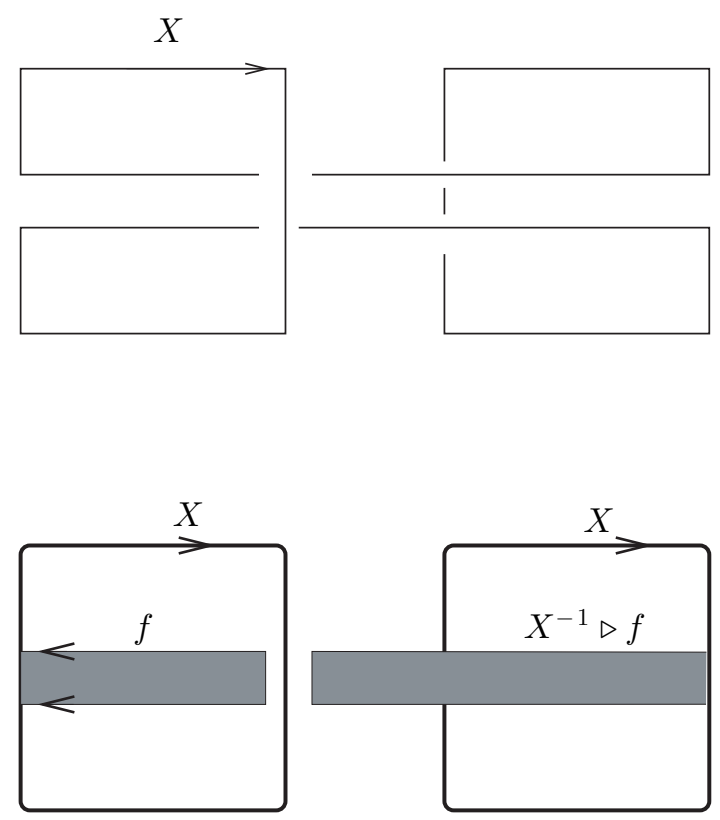

$$
\left\{\begin{array}{c}
\partial(f)=X X^{-1} \\
X^{-1} \triangleright f=1
\end{array}\right.
$$

Figure 18. Calculation of $\Pi_{2}\left(M_{1}, M^{(1)}\right)$. The knot on top is the pre-knot of the knot with bands $K_{1}$ on the bottom.

with the handle decomposition determined by $K_{1}$. The calculation of $\Pi_{2}\left(M_{1}, M_{1}^{(1)}\right)$ appears in Figure 18. This permits us to conclude that

$$
\Pi_{2}\left(M_{1}, M_{1}^{(1)}\right)=\mathcal{U}\left(\{f\} \stackrel{f \mapsto 1}{\longrightarrow} F(X) ; X^{-1} \triangleright f=1\right),
$$

where $F(X)$ is the free group on the variable $X$.

Consider the knot with bands shown in Figure 19, Let $M_{2}$ be the complement of the knotted surface $\Sigma_{2}$ represented by it. From Figure 19 we have

$$
\Pi_{2}\left(M_{2}, M_{2}^{(1)}\right)=\mathcal{U}(\{e\} \stackrel{e \mapsto 1}{\longrightarrow} F(X) ; e=1) .
$$



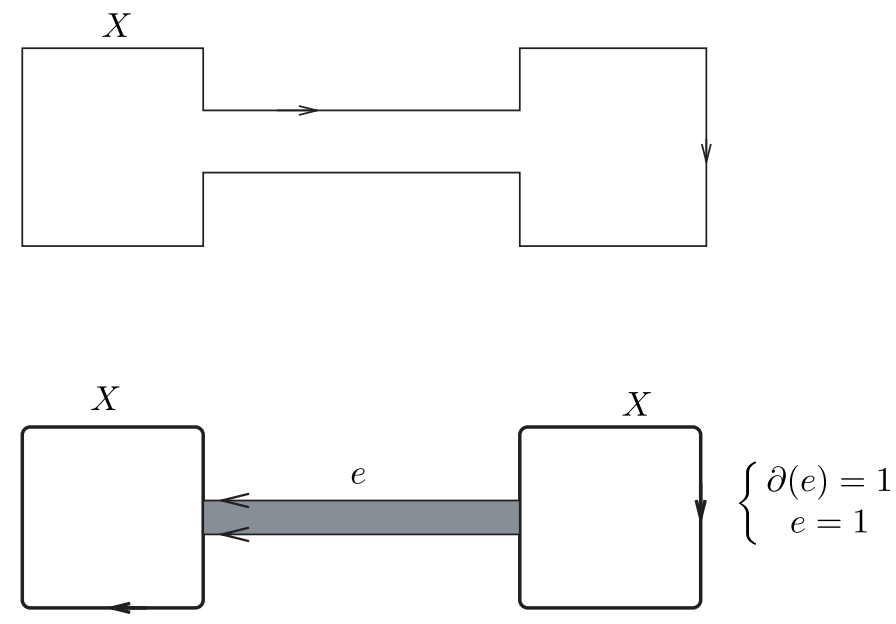

Figure 19. Calculation of $\Pi_{2}\left(M_{2}, M_{2}^{(1)}\right)$. The knot on top is the pre-knot of the knot with bands $K_{2}$ on the bottom.
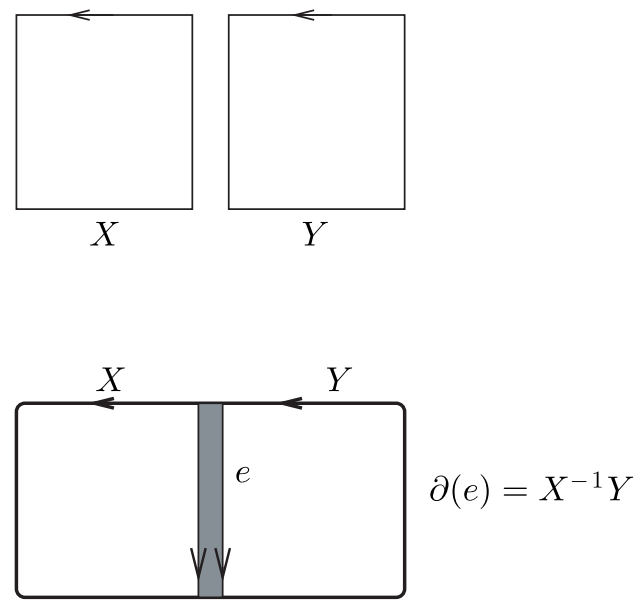

Figure 20. Calculation of $\Pi_{2}\left(M_{3}, M_{3}^{(1)}\right)$. The knot on top is the pre-knot of the knot with bands $K_{3}$ on the bottom.

Likewise, consider the complement $M_{3}$ of the knotted surface $\Sigma_{3}$ represented by the knot with bands $K_{3}$ of figure 20, Then:

$$
\Pi_{2}\left(M_{3}, M_{3}^{(1)}\right)=\mathcal{F}\left(\{e\} \stackrel{e \mapsto X^{-1} Y}{\longrightarrow} F(X, Y)\right) .
$$

Here $F(X, Y)$ is the free group on the variables $X$ and $Y$. Note that in this case we can find a sphere containing the post-knot of $K_{3}$ in the region inside it that does not intersect the unique band of $K_{3}$, hence there are no 2-relations motivated by the attachment of 3-handles; see Theorem 2.6.

2.3. Spun trefoil. A knot with bands representing the Spun Trefoil (a knotted sphere $S^{2}$ embedded in $S^{4}$ ) appears in Figure 21, This can be obtained for example 


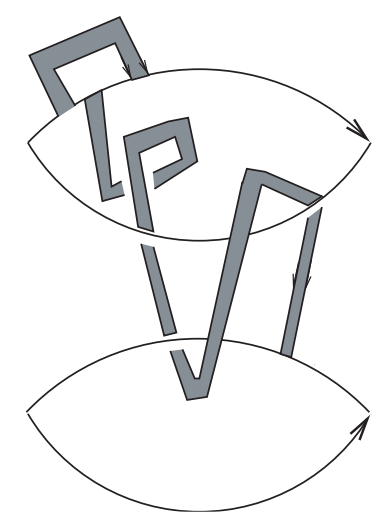

Figure 21. Representing the Spun Trefoil by using a knot with bands.

from the marked vertex diagram of it in [31, Figure 5], by switching the marked vertices to bands, and isotoping the final result so that the post-knot of it is a standard diagram of the unlink.

Let $M=S^{4} \backslash \nu(\Sigma)$ be the complement of the Spun Trefoil $\Sigma$. We display in Figure 22 the calculation of $\Pi_{2}\left(M, M^{(1)}\right)$. This permits us to conclude that $\Pi_{2}\left(M, M^{(1)}\right)$ can be presented by the map $\{e, f\} \rightarrow F(X, Y)$ such that $e \mapsto A^{-1} X$, where $A=X Y X Y X^{-1} Y^{-1} X^{-1}$, and $f \mapsto 1$, also considering the 2-relations

$$
\left(X^{-1} \triangleright f\right)\left(Y^{-1} X^{-1} \triangleright f^{-1}\right)\left(X^{-1} Y^{-1} X^{-1} \triangleright f\right)=1
$$

and

$$
\left(X^{-1} \triangleright f^{-1}\right)\left(X^{-1} Y^{-1} X^{-1} \triangleright f^{-1}\right)\left(Y^{-1} X^{-1} \triangleright f\right)=1 .
$$

Here $F(X, Y)$ is the free group on $X$ and $Y$. Given that $\partial(f)=1$, it follows that all the elements of the form $Z \triangleright f$, where $Z \in \pi_{1}\left(M^{(1)}\right)$, are central in $\pi_{2}\left(M^{(2)}, M^{(1)}\right)$, by the second condition of the definition of crossed modules. In particular the two 2-relations are equivalent; thus we can skip one of them.

See Section 3.2 .2 for calculations in the Spun Hopf Link case.

2.3.1. The second homotopy group of the spun trefoil complement. The discussion of Section 1.1.2 will now be needed. We want to determine the kernel of the map $\pi_{2}\left(M, M^{(1)}\right) \rightarrow \pi_{1}\left(M^{(1)}\right)$, where $M$ is the complement of the Spun Trefoil; cf. (1.2).

Let $K \subset S^{3}=\mathbb{R}^{3} \cup\{\infty\}$ be a knot. Suppose that the projection on the last variable is a Morse function in $K$. Then, similarly with the 4-dimensional case, we have a handle decomposition of the complement of $K$, where minimal points induce 1-handles of the complement and maximal points induce 2-handles. At the end we have to attach one extra 3-handle, which will cancel out one of the 2handles previously attached. Therefore, the complement of the Trefoil Knot, shown in Figure 23, admits a handle decomposition with one 0-handle, two 1-handles and one 2-handle; see [23], exercise 6.2.2. Note that 2-dimensional CW-complexes with a unique 2-cell are classified by their fundamental group, up to homotopy equivalence; see [27.

Consider the CW-complex $N$ with one 0-cell $\{*\}$, two 1-cells $X$ and $Y$ and a 2-cell $e$ attaching along $A^{-1} X$. Here $A=X Y X Y X^{-1} Y^{-1} X^{-1}$. Then $N$ is homotopic to the Trefoil Knot complement. We can prove this for example from 

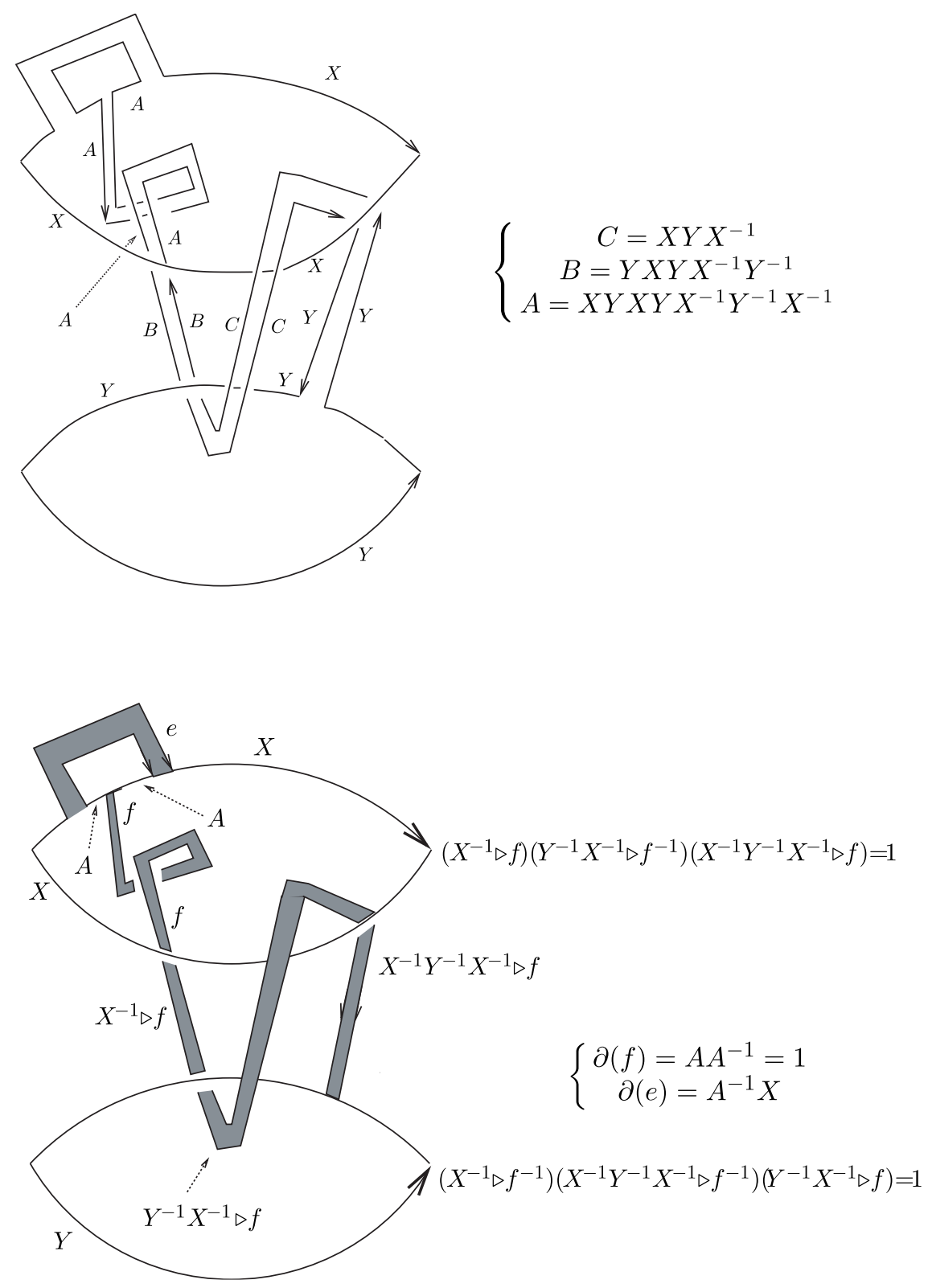

Figure 22. Calculation of $\Pi_{2}\left(M, M^{(1)}\right)$, where $M$ is the complement of the Spun Trefoil.

the fact that $N$ has a unique 2-cell and its fundamental group is isomorphic to the fundamental group of the Trefoil Knot complement. In particular $\pi_{2}(N, *)=\{0\}$, by the well known theorem (due to Papakyriakopoulos) asserting that 3-dimensional (one component) knot complements are aspherical; see 37, 41. On the other hand, we can represent $\pi_{2}(N, *)$ as $\operatorname{ker}\left\{\partial: \pi_{2}\left(N, N^{1}, *\right) \rightarrow \pi_{1}\left(N^{1}, *\right)\right\}$. Note that $\Pi_{2}\left(N, N^{1}, *\right)$ is the free crossed module on the map $e \mapsto A^{-1} X \in F(X, Y)$. 


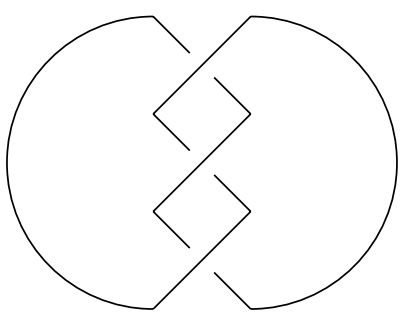

FiguRE 23. Trefoil Knot.

Let $M$ be the complement of the Spun Trefoil, with handle decomposition as above. Let $R^{\prime}$ be the subgroup of $\pi_{2}\left(M^{(2)}, M^{(1)}\right)$ generated by the elements $B \triangleright f$, where $B \in F(X, Y)$. Since $\partial(f)=1$, then (see Section 1.1.2) the group $\pi_{2}\left(M^{(2)}, M^{(1)}\right)$ is the direct sum of $\pi_{2}\left(N, N^{1}, *\right)$ and $R^{\prime}$. Moreover, $R^{\prime}$ is the free abelian module with basis $\{f\}$ over the group algebra of $\pi_{1}(M)=\operatorname{coker}(\partial) \doteq G$, where $\partial: \pi_{2}\left(N, N^{1}, *\right) \rightarrow \pi_{1}\left(N^{1}, *\right)=F(X, Y)$, with the obvious action of $F(X, Y)$. In addition, we have that $G=F(X, Y) /\left\langle X A^{-1}=1\right\rangle$, which is isomorphic with the fundamental group of the complement of the Trefoil Knot.

Now, $\left(X^{-1} \triangleright f\right)-\left(Y^{-1} X^{-1} \triangleright f\right)+\left(X^{-1} Y^{-1} X^{-1} \triangleright f\right) \in R^{\prime}$ (note that we switched to additive notation). Consider the abelian $\mathbb{Z}(G)$-module:

$$
R=\frac{R^{\prime}}{\left\langle\left(X^{-1} \triangleright f\right)-\left(Y^{-1} X^{-1} \triangleright f\right)+\left(X^{-1} Y^{-1} X^{-1} \triangleright f\right)=0\right\rangle} .
$$

Then it follows that $\pi_{2}\left(M, M^{(1)}\right)$, as an $F(X, Y)$-module, is the direct sum of $R$ and $\pi_{2}\left(N, N^{1}, *\right)$.

The CW-complex $N$ is aspherical; thus the kernel of the boundary map of $\Pi_{2}\left(M, M^{(1)}\right)$ when restricted to $\pi_{2}\left(N, N^{1}, *\right)$ is trivial. In particular, $\pi_{2}(M)=$ $\operatorname{ker}\left\{\partial: \pi_{2}\left(M, M^{(1)}\right) \rightarrow \pi_{1}\left(M^{(1)}\right)\right\}=R$. It follows that the second homotopy group of the complement of the Spun Trefoil is the free abelian module over $\mathbb{Z}(G)$ with one generator $f$ and the relation $\left(X^{-1} \triangleright f\right)-\left(Y^{-1} X^{-1} \triangleright f\right)+\left(X^{-1} Y^{-1} X^{-1} \triangleright f\right)=0$. This is consistent with the calculation in 31.

\section{The "CROSSED MOdUle inVARIANT" OF HOMOTOPY TYPeS}

3.1. Definition of the invariant. Let $M$ be a CW-complex. As we have seen in Section 1.2.2, the fundamental crossed module $\Pi_{2}\left(M, M^{1}\right)$ depends only on the homotopy type of $M$, as a space, up to free products (in the category of crossed modules) with $\Pi_{2}\left(D^{2}, S^{1}\right)$. Given a cell decomposition of $M$, it is in principle possible to obtain a presentation of $\Pi_{2}\left(M, M^{1}\right)$. However, it may be difficult to distinguish between two crossed modules (up to free products with $\Pi_{2}\left(D^{2}, S^{1}\right)$ ) presented this way.

It is possible in some cases to distinguish between finitely generated groups by using the Alexander's Invariant (see [15]), or by counting the number of morphisms from them into a finite group. The latter method can also be used, with due adaptations, in the case of finitely generated crossed modules. We have:

Theorem 3.1. Let $M$ be a finite $C W$-complex with a unique 0 -cell, which we take to be its base point $*$. Let $\mathcal{G}=(G, E, \partial, \triangleright)$ be a finite crossed module. Here $\partial: E \rightarrow G$ is a group morphism and $\triangleright$ is a left action of $G$ on $E$ by automorphisms. The 
quantity

$$
I_{\mathcal{G}}(M)=\frac{\# \operatorname{Hom}\left(\Pi_{2}\left(M, M^{1}\right), \mathcal{G}\right)}{(\# E)^{b_{1}\left(M^{1}\right)}}
$$

is finite, does not depend on the $C W$-decomposition of $M$ and is a homotopy invariant of $M$, as a space. Here $b_{1}\left(M^{1}\right)$ denotes the first Betti number of the 1-skeleton $M^{1}$ of $M$.

We call this homotopy invariant of CW-complexes the "Crossed Module Invariant".

Proof. The finiteness of $I_{\mathcal{G}}(M)$ follows from the fact (Theorem 1.10) that if $M$ is finite, then the group $\pi_{2}\left(M, M^{1}\right)$ is finitely generated as a module over $\pi_{1}\left(M^{1}\right)$, itself a finitely generated group.

Let $N$ be a CW-complex with a unique 0 -cell $*^{\prime}$, such that $N$ is homotopic to $M$ as a space. By the discussion in Section 1.2.2, there exist positive integers $m$ and $n$ such that

$$
\Pi_{2}\left(M, M^{1}, *\right) \vee \Pi_{2}\left(D^{2}, S^{1}, *\right)^{\vee n} \cong \Pi_{2}\left(N, N^{1}, *^{\prime}\right) \vee \Pi_{2}\left(D^{2}, S^{1}, *^{\prime}\right)^{\vee m} .
$$

By using the universal property defining free products of crossed modules (Example 1.3) it follows that

$$
\begin{aligned}
\# \operatorname{Hom}\left(\Pi_{2}\left(M, M^{1}, *\right), \mathcal{G}\right) \times\left(\# \operatorname{Hom}\left(\Pi_{2}\left(D^{2}, S^{1}, *\right), \mathcal{G}\right)\right)^{n} \\
=\# \operatorname{Hom}\left(\Pi_{2}\left(N, N^{1}, *\right), \mathcal{G}\right) \times\left(\# \operatorname{Hom}\left(\Pi_{2}\left(D^{2}, S^{1}, *\right), \mathcal{G}\right)\right)^{m} .
\end{aligned}
$$

Since $\Pi_{2}\left(D^{2}, S^{1}, *\right)=(\mathbb{Z}, \mathbb{Z}$, id,$\triangleright)$, where $\triangleright$ is the trivial action, we have that $\# \operatorname{Hom}\left(\Pi_{2}\left(D^{2}, S^{1}, *\right), \mathcal{G}\right)=\# E$. The result follows from the fact that we necessarily have $b_{1}\left(M^{1}\right)+n=b_{1}\left(N^{1}\right)+m$; see the comments after Corollary 1.12 .

Let $M$ be a compact manifold with a handle decomposition. In this case the crossed module invariant $I_{\mathcal{G}}(M)$ can be calculated by using the crossed module $\Pi_{2}\left(M, M^{(1)}\right)$, where as before $M^{(1)}$ is the 1-handlebody of $M$, made out of the 0 and 1-handles of $M$. This follows immediately from the discussion at the beginning of the previous chapter.

The Crossed Module Invariant $I_{\mathcal{G}}$ has the following geometric interpretation; see [18, 20]. Let $\mathcal{G}$ be a finite crossed module. Then $\mathcal{G}$ has a classifying space $B_{\mathcal{G}}$ with a natural base point $*$; see $\left[8,4\right.$. Consider the space $\operatorname{TOP}\left((M, *),\left(B_{\mathcal{G}}, *\right)\right)$ of continuous maps $(M, *) \rightarrow\left(B_{\mathcal{G}}, *\right)$, provided with the $k$-ification of the compactopen topology. Then

$$
I_{\mathcal{G}}(M)=\sum_{f \in\left[(M, *),\left(B_{\mathcal{G}}, *\right)\right]} \frac{1}{\# \pi_{1}\left(\operatorname{TOP}\left((M, *),\left(B_{\mathcal{G}}, *\right)\right), f\right)} .
$$

Here $\left[(M, *),\left(B_{\mathcal{G}}, *\right)\right]=\pi_{0}\left(\operatorname{TOP}\left((M, *),\left(B_{\mathcal{G}}, *\right)\right)\right)$ denotes the set of pointed homotopy classes of maps $(M, *) \rightarrow\left(B_{\mathcal{G}}, *\right)$.

The results in this subsection extend in a natural way to crossed complexes; see [18. 
3.1.1. Relation with algebraic 2-types. Recall that a 2-type is a path-connected topological space $M$ such that $\pi_{k}(M)=\{0\}, \forall k>2$. If $M$ is a connected CWcomplex with a base point $*$ which is a 0 -cell, then $T_{2}(M)$ is the based cellular space defined from $M^{3}$ by killing all the homotopy groups $\pi_{k}(M)$ of $M$ with $k>2$ in the usual way; see for example [25. Example 4.17]. It is well known that $T_{2}(M)$ does not depend on the CW-decomposition of $M$ up to homotopy equivalence. If $M$ is a connected CW-complex, then the CW-complex $T_{2}(M)$ is called the topological 2-type of $M$, or, more commonly, the second Postnikov section of $M$.

Let $M$ be a path-connected topological space. Then the algebraic 2-type of $M$ is given by the triple

$$
A_{2}(M)=\left(\pi_{1}(M), \pi_{2}(M), k(M)\right),
$$

where $k \in H^{3}\left(\pi_{1}(M), \pi_{2}(M)\right)$ is the $k$-invariant (or first Postnikov invariant) of $M$; see [32, 16, 33. If $M$ and $N$ are $C W$-complexes, then their topological 2types (up to homotopy) coincide if and only if their algebraic 2-types coincide (up to isomorphism); see [1, 16, 30]. On the other hand we have that $\Pi_{2}\left(M, M^{1}\right)=$ $\Pi_{2}\left(M^{3}, M^{1}\right)=\Pi_{2}\left(T_{2}(M), T_{2}(M)^{1}\right)$, where both equalities follow from the Cellular Approximation Theorem. Therefore, we have:

Theorem 3.2. Let $\mathcal{G}$ be a finite crossed module. The homotopy invariant $I_{\mathcal{G}}(M)$ depends only on the (algebraic or topological) 2-type of $M$.

Therefore a natural issue is how useful the Crossed Module Invariant is for separating 2-types.

3.2. Applications to knotted surfaces. Theorem 3.1 tells us that we can define invariants of knotted surfaces by considering the invariants $I_{\mathcal{G}}$ of their complements in $S^{4}$, where $\mathcal{G}$ is a finite crossed module. The previous chapter provides us with an algorithm for this type of calculation.

Recall the construction of a crossed module presented by a map, with 2-relations, in Section 1.1. It follows immediately that:

Lemma 3.3. Suppose that $G$ is a free group, say on the set $L$. Thus if $G^{\prime}$ is a group, then any map $\phi_{0}: L \rightarrow G^{\prime}$ extends uniquely to a group morphism $\phi: G \rightarrow G^{\prime}$. Let $K=\left\{m_{1}, \ldots, m_{k}\right\}$ be a set provided with a map $\partial_{0}: K \rightarrow G$. Also let $\left\{r_{1}, \ldots, r_{n}\right\}$ be a set of 2-relations in the free crossed module on the map $\partial_{0}: K \rightarrow G$. Let $\mathcal{G}^{\prime}=\left(G^{\prime}, E^{\prime}, \partial^{\prime}, \triangleright^{\prime}\right)$ be a crossed module. There exists a one-to-one correspondence between crossed module maps

$$
\mathcal{U}\left(K \stackrel{\partial_{0}}{\longrightarrow} G ; r_{1}, \ldots, r_{n}=1\right) \rightarrow \mathcal{G}^{\prime}
$$

and pairs of maps $\phi_{0}: L \rightarrow G^{\prime}$ and $\psi_{0}: K \rightarrow E^{\prime}$ satisfying:

(1) $\left(\phi \circ \partial_{0}\right)\left(m_{i}\right)=\left(\partial^{\prime} \circ \psi_{0}\right)\left(m_{i}\right) ; i=1, \ldots, k$,

(2) $\psi\left(r_{i}\right)=1_{E^{\prime}}, i=1, \ldots, n$.

Here $(\phi, \psi)$ is the map $\mathcal{F}\left(\partial_{0}: K \rightarrow G\right) \rightarrow \mathcal{G}^{\prime}$ determined by $\phi_{0}$ and $\psi_{0}$. In particular if

$$
r=\left(X_{1}, m_{1}\right)^{\theta_{1}}\left(X_{2}, m_{2}\right)^{\theta_{2}} \ldots\left(X_{l}, m_{l}\right)^{\theta_{l}}
$$

where $X_{i} \in G, m_{i} \in K$ and $\theta_{i} \in \mathbb{Z}$, for $i=1,2, \ldots l$, then

$$
\psi(r)=\left(\phi\left(X_{1}\right) \triangleright^{\prime} \psi_{0}\left(m_{1}\right)\right)^{\theta_{1}}\left(\phi\left(X_{2}\right) \triangleright^{\prime} \psi_{0}\left(m_{2}\right)\right)^{\theta_{2}} \ldots\left(\phi\left(X_{l}\right) \triangleright^{\prime} \psi_{0}\left(m_{l}\right)\right)^{\theta_{l}} \in E^{\prime} .
$$


As an example, consider the knotted surface complements $M_{1}, M_{2}$ and $M_{3}$ of Section 2.2.4. By the previous lemma it follows that

$$
\begin{aligned}
& I_{\mathcal{G}}\left(M_{1}\right)=\frac{\#\left\{X \in G, f \in E \mid \partial(f)=1, X^{-1} \triangleright f=1\right\}}{\# E}=\frac{\# G}{\# E}, \\
& I_{\mathcal{G}}\left(M_{2}\right)=\frac{\#\{X \in G, e \in E \mid \partial(e)=1, e=1\}}{\# E}=\frac{\# G}{\# E}, \\
& I_{\mathcal{G}}\left(M_{3}\right)=\frac{\#\left\{X, Y \in G, e \in E \mid \partial(e)=X^{-1} Y\right\}}{\# E^{2}}=\frac{\# G}{\# E} .
\end{aligned}
$$

In fact all these knotted surfaces are isotopic to the trivial knotted sphere.

Suppose that $\mathcal{G}=(G, E, \partial, \triangleright)$ is a finite crossed module with $G$ abelian and that $\partial=1_{G}$, from which it follows that $E$ is abelian. Then if $M$ is the complement of the Spun Trefoil it follows that

$$
I_{\mathcal{G}}(M)=\frac{\#\left\{(X, f) \in G \times E:(X \triangleright f)\left(X^{2} \triangleright f^{-1}\right)\left(X^{3} \triangleright f\right)\right\}}{\# E} .
$$

This agrees with the calculation in [17.

This information is sufficient for proving that the Spun Trefoil is knotted. For example, consider the crossed module $\mathcal{A}=\left(\mathbb{Z}_{2}, \mathbb{Z}_{3}, \partial, \triangleright\right)$, where $\mathbb{Z}_{2}=\{+1,-1\}$, with the trivial boundary map $\mathbb{Z}_{3} \rightarrow \mathbb{Z}_{2}$. The action of $\mathbb{Z}_{2}=\{+1,-1 ; \times\}$ in $\mathbb{Z}_{3}=\{[0],[1],[2] ;+\}$ is $a \triangleright v=a v$. Then $I_{\mathcal{A}}(M)=4 / 3$. However, if $M_{1}$ is the complement of the trivial knotted sphere, then $I_{\mathcal{A}}\left(M_{1}\right)=2 / 3$.

Notice that we would not have been able to prove this (known) fact if we had used only the fundamental group of the complement of the Spun Trefoil and had counted the number of morphisms from it into an abelian group, since the first homology group of a knotted surface complement depends only on the intrinsic topology of the knotted surface, and not on the embedding. See also Remark 3.6 and Section 3.2.3.

3.2.1. A general algorithm. Let $\mathcal{G}=(G, E, \partial, \triangleright)$ be a finite crossed module and let $M=S^{4} \backslash \nu(\Sigma)$ be the complement of the knotted surface $\Sigma$. To calculate $I_{\mathcal{G}}(M)$ we do not need to determine $\Pi_{2}\left(M, M^{(1)}\right)$ fully, which, since $\mathcal{G}$ verifies relations of its own, can be much more complicated than calculating $I_{\mathcal{G}}(M)$ alone. To this end we define:

Definition 3.4 ( $\mathcal{G}$-coloring). Let $K$ be a knot with bands representing some oriented knotted surface $\Sigma$. As usual, we suppose that $K$ is provided with a regular projection as before, such that the post-knot of $K$ is a standard diagram of the unlink and such that each band of $K$ always has the same side facing upwards. Let $\mathcal{G}=(G, E, \partial, \triangleright)$ be a finite crossed module. A $\mathcal{G}$-coloring of $K$ is an assignment of an element of $G$ to each arc of the pre-knot $K_{-}$of $K$ and an element of $E$ to each arc of the bands of $K$, satisfying the conditions of Figures 12, 13, 15] and 17. These conditions should be interpreted in light of Theorem 2.6 .

Note that the additional relations obtained when a thin component of $K$ passes under a fat component are dealt with by the Wirtinger relations for $K_{-}$. 
By Lemma 3.3 it follows that:

Theorem 3.5. Let $\Sigma \subset S^{4}$ be an oriented knotted surface, and choose a knot with bands $K$ representing $\Sigma$, as well as a regular projection of $K$. Suppose that the post-knot of $K$ is a standard diagram of the unlink, and that each band of $K$ always has the same side facing upwards. Let $\mathcal{G}=(G, E, \partial, \triangleright)$ be a finite crossed module. We have

$$
I_{\mathcal{G}}\left(S^{4} \backslash \nu(\Sigma)\right)=\frac{\#\{\mathcal{G} \text {-colorings of } K\}}{\# E^{\left.\# \text { circles of } K_{-}\right\}}} .
$$

Note that, given a knot with bands $K$ representing the knotted surface $\Sigma \subset S^{4}$, the handle decomposition of the complement of $\Sigma$ in $S^{4}$ constructed from $K$ has a unique 0 -handle and a 1-handle for each circle of the pre-knot $K_{-}$of $K$.

The formula for $I_{\mathcal{G}}\left(S^{4} \backslash \nu(\Sigma)\right)$ provided by the previous theorem simplifies considerably when $\mathcal{G}=(G, E, \partial, \triangleright)$ is a crossed module with $G$ abelian and $\partial=1_{G}$, which implies $E$ is abelian. In this case, for calculation purposes, all strands of a knot with bands $K$ can pass through each other, except when one is a thin component and the other is a band. In addition, the coloring of an arc of $K_{-}$depends only on the component of $K_{-}$to which it belongs; see Figures 12]13, 15, and 17. and adapt them to the case when $G$ is abelian and $\partial=1_{G}$.

Remark 3.6. We prove in [19] that the Crossed Module Invariant $I_{\mathcal{G}}$ is strong enough to distinguish some pairs $\left(\Sigma, \Sigma^{\prime}\right)$ of non-isotopic knotted surfaces with the same fundamental group of the complement, and with $\Sigma$ diffeomorphic with $\Sigma^{\prime}$ as 2-dimensional manifolds; see also Section 3.2.3. In 38, it was asserted the existence of pairs $\Sigma, \Sigma^{\prime} \subset S^{4}$ of knotted spheres such that $\pi_{1}\left(S^{4} \backslash \nu(\Sigma)\right) \cong \pi_{1}\left(S^{4} \backslash \nu\left(\Sigma^{\prime}\right)\right)$ and $\pi_{2}\left(S^{4} \backslash \nu(\Sigma)\right) \cong \pi_{2}\left(S^{4} \backslash \nu\left(\Sigma^{\prime}\right)\right)$, as modules over $\pi_{1}\left(S^{4} \backslash \nu(\Sigma)\right)=\pi_{1}\left(S^{4} \backslash\right.$ $\left.\nu\left(\Sigma^{\prime}\right)\right)$, but with $k\left(S^{4} \backslash \nu(\Sigma)\right) \neq k\left(S^{4} \backslash \nu\left(\Sigma^{\prime}\right)\right)$. Since the invariant $I_{\mathcal{G}}(M)$ depends only on the algebraic 2-type of $M$ (see Section 3.1.1), it would be interesting to determine whether $I_{\mathcal{G}}$ is strong, and practical, enough to distinguish between pairs of embedded spheres with this property, completing the results of [19].

3.2.2. Spun Hopf Link. Consider the Spun Hopf Link $\Sigma$ obtained by spinning the Hopf Link depicted in Figure 24. Therefore $\Sigma$ is an embedding of a disjoint union of two tori $S^{1} \times S^{1}$ into $S^{4}$. A knot with bands $K$ representing the Spun Hopf Link appears in Figure 25 .

Let us calculate $\Pi_{2}\left(M, M^{(1)}\right)$, where $M$ is the complement of the Spun Hopf Link, with the handle decomposition determined by $K$. This calculation appears in Figure 26. This permits us to conclude that $\Pi_{2}\left(M, M^{(1)}\right)$ is the crossed module presented by the map $\{e, f, g, h\} \rightarrow F(X, Y)$, where $f, h \mapsto 1, e \mapsto X^{-1} Y X Y^{-1}$ and $g \mapsto X^{-1} Y^{-1} X Y$, considering the 2-relations

$$
f\left(X \triangleright f^{-1}\right) h\left(Y \triangleright h^{-1}\right)=1
$$

and

$$
(X \triangleright f) f^{-1}(Y \triangleright h) h^{-1}=1 .
$$

As usual, $F(X, Y)$ is the free group on the variables $X$ and $Y$. These 2-relations are equivalent, thus we can skip one of them. To prove this we need to use the fact that $\partial(h), \partial(f)=1$, which implies that both $h$ and $f$ are central in $\pi_{2}\left(M^{(2)}, M^{(1)}\right)$.

Let $\mathcal{G}=(G, E, \partial, \triangleright)$ be a finite crossed module. We can easily calculate $I_{\mathcal{G}}\left(S^{4} \backslash \nu(\Sigma)\right)$, for $\Sigma$ the Spun Hopf Link, by using this calculation. Suppose that 


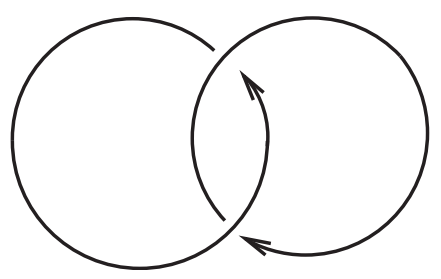

FiguRE 24. Hopf Link.

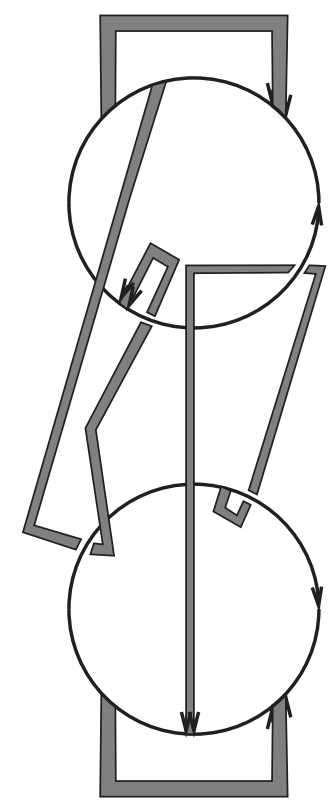

Figure 25. A knot with bands representing the Spun Hopf Link.

$\mathcal{G}=(G, E, \partial, \triangleright)$ is a finite crossed module with $G$ abelian and $\partial=1_{G}$. We have

$$
I_{\mathcal{G}}\left(S^{4} \backslash \nu(\Sigma)\right)=\#\left\{X, Y \in G ; f, h \in E \mid f\left(X \triangleright f^{-1}\right) h\left(Y \triangleright h^{-1}\right)=1_{E}\right\} .
$$

This particularizes to $I_{\mathcal{A}}\left(S^{4} \backslash \nu(\Sigma)\right)=18$, for the case when $\mathcal{A}=\left(\mathbb{Z}_{2}, \mathbb{Z}_{3}, \partial, \triangleright\right)$ is the crossed module defined in Section 3.2 .

If $T$ is the trivial embedding of a disjoint union of two tori we have, for any finite crossed module $\mathcal{G}=(G, E, \partial, \triangleright)$,

$$
I_{\mathcal{G}}\left(S^{4} \backslash \nu(T)\right)=\left(\frac{(\# G)(\# \operatorname{ker} \partial)^{2}}{\# E}\right)^{2},
$$

which specializes to

$$
I_{\mathcal{G}}\left(S^{4} \backslash \nu(T)\right)=(\# G)^{2}(\# E)^{2}
$$

in the particular case for which $G$ is abelian and $\partial=1_{G}$. Comparing this with the value for $I_{\mathcal{A}}\left(S^{4} \backslash \nu(\Sigma)\right)$ when $\Sigma$ is the Spun Hopf Link proves that $\Sigma$ is knotted.

We can also determine the second homotopy group of the complement $M$ of the Spun Hopf Link from the presentation of the fundamental crossed module 

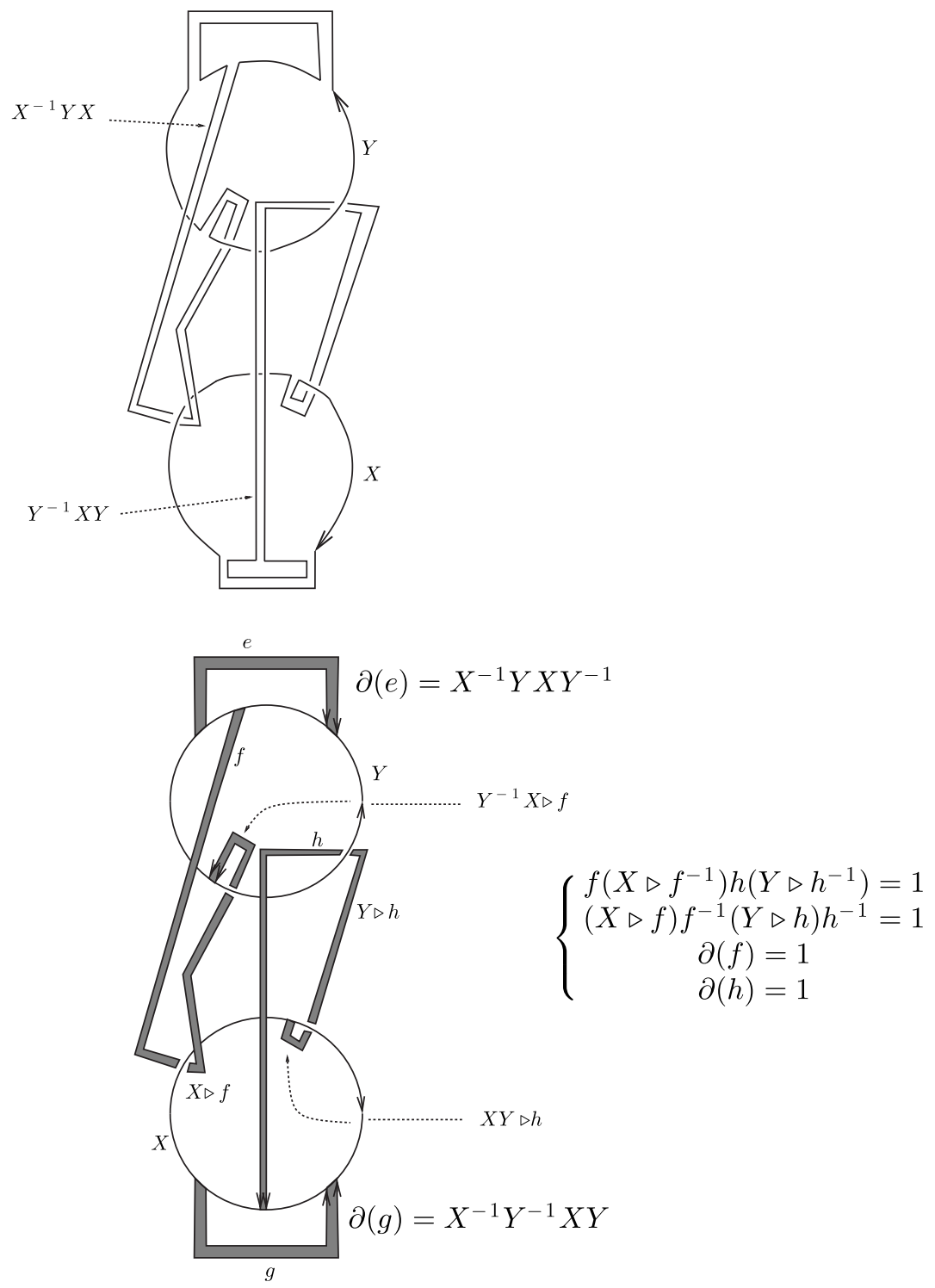

Figure 26. Calculation of $\Pi_{2}\left(M, M^{(1)}\right)$, where $M=S^{4} \backslash \nu(\Sigma)$. Here $\Sigma$ is the Spun Hopf Link.

$\Pi_{2}\left(M, M^{(1)}\right)$. Proceeding as in the case of the Spun Trefoil, it follows that $\pi_{2}(M)$ is the quotient of the free abelian module over the group algebra of $G=\{X, Y \mid X Y=$ $Y X\}$ (the fundamental group of $M$ ) with generators $\{z, f, h\}$ by the relation $X \triangleright$ $f-f+(Y \triangleright h)-h=0$.

To prove this, we need to use the fact that the CW-complex $N$ constructed with two 1-cells $X$ and $Y$ and two 2-cells $e$ and $g$, attaching in a way such that $\partial(e)=X^{-1} Y X Y^{-1}$, whereas $\partial(g)=X^{-1} Y^{-1} X Y$, is such that $\pi_{1}(N)=G$ and 


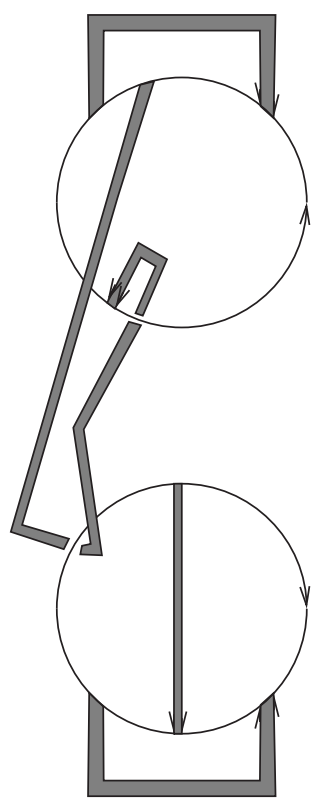

FiguRe 27. A knot with bands $K^{\prime}$ representing the knotted surface $\Sigma^{\prime}$

$\pi_{2}(N)$ is the free abelian module over $\mathbb{Z}(G)$ with basis $\{z\}$. This follows from the fact that $N \cong T^{2} \vee S^{2}$, easy to prove. Here $T^{2}=S^{1} \times S^{1}$ is the torus.

3.2.3. Final example. Consider the knotted surface $\Sigma^{\prime}$ obtained from the knot with bands $K^{\prime}$ which appears in Figure 27. Similarly with the Spun Hopf Link, this knotted surface is, topologically, diffeomorphic with the disjoint union of two tori $S^{1} \times S^{1}$.

Let us determine $\Pi_{2}\left(N, N^{(1)}\right)$, where $N=S^{4} \backslash \nu\left(\Sigma^{\prime}\right)$, with the handle decomposition determined by $K^{\prime}$. This calculation appears in Figure 28. This permits us to conclude that $\Pi_{2}\left(N, N^{(1)}\right)$ is the crossed module presented by the map $\{e, f, g, h\} \rightarrow F(X, Y)$, where $f, h, g \mapsto 1$ and $e \mapsto X^{-1} Y X Y^{-1}$, considering the 2-relation $f\left(X \triangleright f^{-1}\right)=1$. Recall that $F(X, Y)$ is the free group on the variables $X$ and $Y$.

Let $\mathcal{G}=(G, E, \partial, \triangleright)$ be a finite crossed module. By using this calculation, we can determine $I_{\mathcal{G}}\left(S^{4} \backslash \nu\left(\Sigma^{\prime}\right)\right)$. Suppose that $\mathcal{G}=(G, E, \partial, \triangleright)$ is a finite crossed module with $G$ abelian and $\partial=1_{G}$. We have

$$
I_{\mathcal{G}}\left(S^{4} \backslash \nu\left(\Sigma^{\prime}\right)\right)=\# G \# E \#\left\{X \in G ; f \in E \mid f\left(X \triangleright f^{-1}\right)=1_{E}\right\} .
$$

This particularizes to $I_{\mathcal{A}}\left(S^{4} \backslash \nu\left(\Sigma^{\prime}\right)\right)=24$, for the case when $\mathcal{A}=\left(\mathbb{Z}_{2}, \mathbb{Z}_{3}, \partial, \triangleright\right)$ is the crossed module defined in Section 3.2. This in particular proves that $\Sigma^{\prime}$ is knotted and that $\Sigma^{\prime}$ is not isotopic to the Spun Hopf Link $\Sigma$. 

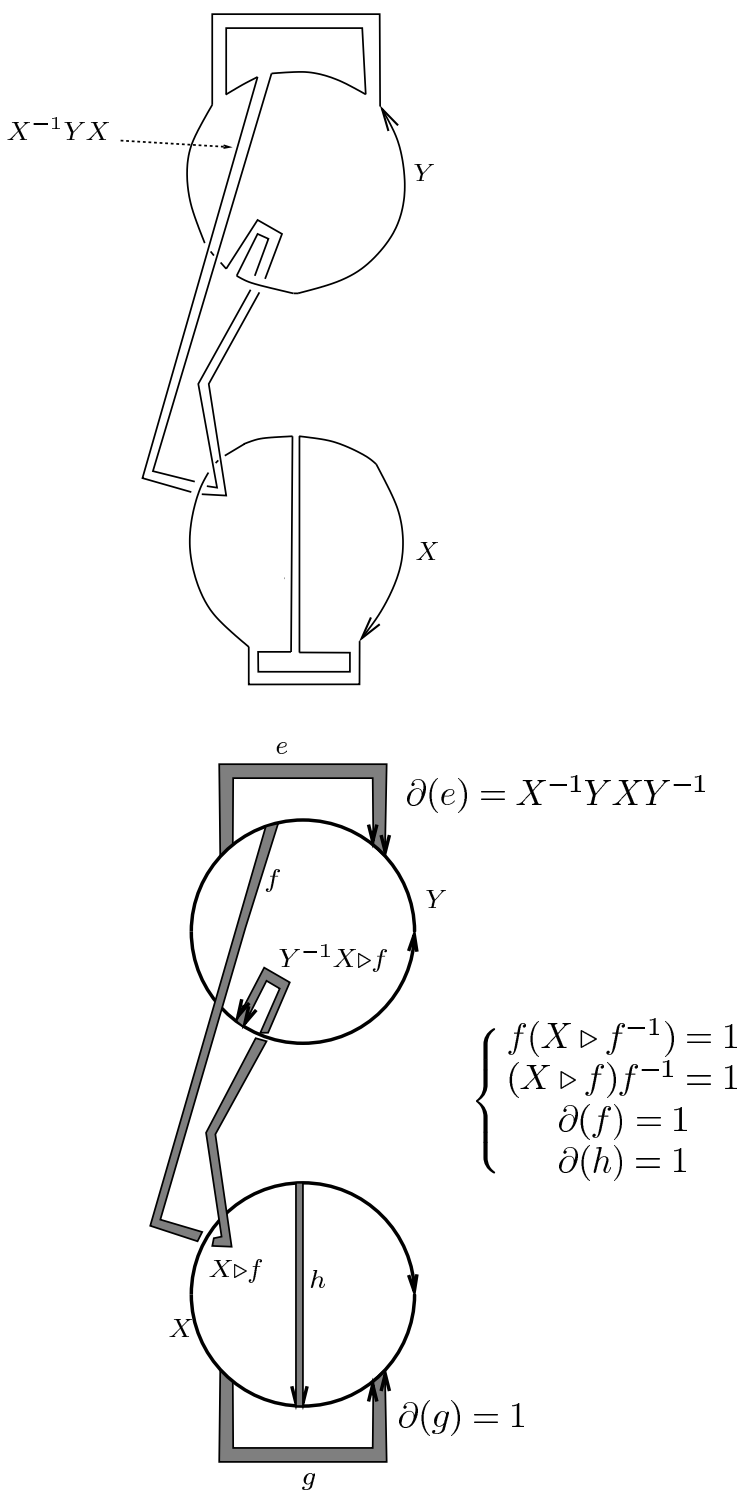

Figure 28. Calculation of $\Pi_{2}\left(N, N^{(1)}\right)$, where $N=S^{4} \backslash \nu\left(\Sigma^{\prime}\right)$. Here $\Sigma^{\prime}$ is represented by the knot with bands $K^{\prime}$ of Figure 27.

Note that the fundamental groups of the complements of $\Sigma$ and $\Sigma^{\prime}$ each are isomorphic with $\mathbb{Z} \oplus \mathbb{Z}$ (this can be inferred from the presentations of the fundamental crossed modules of them). Therefore $\Sigma$ and $\Sigma^{\prime}$ are two knotted surfaces (each a disjoint union of two tori $S^{1} \times S^{1}$ ) with the same fundamental group of the complement, but distinguished by their crossed module invariants. This example appears in [19. 


\section{ACKNOWLEDGEMENTS}

The author would like to thank a referee of a previous version of this work, as well as Gustavo Granja, Scott Carter and Roger Picken for useful comments.

\section{REFERENCES}

1. Baues H.J.: Combinatorial Homotopy and 4-Dimensional Complexes. With a preface by Ronald Brown, de Gruyter Expositions in Mathematics, 2, Walter de Gruyter \& Co., Berlin, 1991. MR1096295 (92h:55008)

2. Brown R.A.: Generalized Group Presentation and Formal Deformations of CW-Complexes, Trans. Amer. Math. Soc. 334 (1992), no. 2, 519-549. MR1153010 (93h:57001)

3. Brown K.S.: Cohomology of Groups, Corrected reprint of the 1982 original, Graduate Texts in Mathematics, 87, Springer-Verlag, New York, 1994. MR1324339 (96a:20072)

4. Brown R.: On the Second Relative Homotopy Group of an Adjunction Space: an Exposition of a Theorem of J. H. C. Whitehead, J. London Math. Soc. (2) 22 (1980), no. 1, 146-152. MR579818 (81g:55014)

5. Brown R.: Groupoids and Crossed Objects in Algebraic Topology, Homology Homotopy Appl. 1 (1999), 1-78 (electronic). MR.1691707 (2000d:55002)

6. Brown R.: Crossed Complexes and Homotopy Groupoids as non Commutative Tools for Higher Dimensional Local-to-Global Problems, Galois theory, Hopf algebras, and Semiabelian Categories, 101-130, Fields Inst. Commun., 43, Amer. Math. Soc., Providence, RI, 2004. MR2075583 (2005f:18001)

7. Brown R., Higgins P.J.: On the Connection Between the Second Relative Homotopy Groups of Some related Spaces, Proc. London Math. Soc. (3) 36 (1978), no. 2, 193-212. MR0478150 $(57: 17639)$

8. Brown R., Higgins P.J.: The Classifying Space of a Crossed Complex, Math. Proc. Cambridge Philos. Soc. 110 (1991), no. 1, 95-120. MR.1104605 (92b:55024)

9. Brown R., Higgins P.J.: Colimit Theorems for Relative Homotopy Groups, J. Pure Appl. Algebra 22 (1981), no. 1, 11-41. MR621285 (82m:55015b)

10. Brown R., Higgins P.J., Sivera R.: Nonabelian algebraic topology (in preparation). Part I downloadable.

11. Brown R., Huebschmann J.: Identities Among Relations, Low-Dimensional Topology (Bangor, 1979), pp. 153-202, London Math. Soc. Lecture Note Ser., 48, Cambridge Univ. Press, Cambridge, New York, 1982. MR662431 (83h:57008)

12. Carter S., Kamada S., Saito M.: Surfaces in 4-Space, Encyclopaedia of Mathematical Sciences, 142, Low-Dimensional Topology, III. Springer-Verlag, Berlin, 2004. MR2060067|(2005e:57065)

13. Carter S., Rieger J., Saito M.: A Combinatorial Description of Knotted Surfaces and their Isotopies, Adv. Math. 127 (1997), no. 1, 1-51. MR1445361 (98c:57023)

14. Carter J.C., Saito M.: Knotted Surfaces and their Diagrams, Mathematical Surveys and Monographs, 55, American Mathematical Society, Providence, RI, 1998. MR.1487374 (98m:57027)

15. Crowell R.H., Fox R.H.: Introduction to Knot Theory. Reprint of the 1963 original. Graduate Texts in Mathematics, No. 57, Springer-Verlag, New York, Heidelberg, 1977. MR0445489 (56:3829)

16. Eilenberg S., Mac Lane S.: Determination of the Second Homology and Cohomology Groups of a Space by Means of Homotopy Invariants, Proc. Nat. Acad. Sci. U. S. A. 32 (1946), 277-280. MR0019307 (8:398b)

17. Faria Martins J.: Categorical Groups, Knots and Knotted Surfaces, J. Knot Theory Ramifications 16 (2007), no. 9, 1181-1217. MR2375821

18. Faria Martins J.: On the Homotopy Type and the Fundamental Crossed Complex of the Skeletal Filtration of a CW-Complex, Homology Homotopy and Applications, vol. 9 (2007), no. 1, pp. 295-329. MR2299802

19. Faria Martins J., Kauffman L.: Invariants of Welded Virtual Knots via Crossed Module Invariants of Knotted Surfaces, Compos. Math. 144 (2008), no. 4, 1046-1080. MR 2441256

20. Faria Martins J., Porter T.: On Yetter's Invariant and an Extension of the Dijkgraaf-Witten Invariant to Categorical Groups, Theory and Applications of Categories, vol. 18, 2007, no. 4, pp. 118-150. MR 2299797 (2008a:18010) 
21. Fox R.H.: A Quick Trip Through Knot Theory, 1962, Topology of 3-manifolds and related topics (Proc. The Univ. of Georgia Institute, 1961) pp. 120-167, Prentice-Hall, Englewood Cliffs, N.J. MR0140099 (25:3522)

22. Gordon C. McA.: Homology of Groups of Surfaces in the 4-Sphere, Math. Proc. Cambridge Philos, Soc. 89 (1981), no. 1, 113-117. MR591977|(83d:57016)

23. Gompf R.E., Stipsicz A.I.: 4-Manifolds and Kirby Calculus, Graduate Studies in Mathematics, 20. American Mathematical Society, Providence, RI, 1999. MR1707327 (2000h:57038)

24. Gutiérrez M., Hirschhorn P.: Free Simplicial Groups and the Second Relative Homotopy Group of an Adjunction Space, J. Pure Appl. Algebra 39 (1986), no. 1-2, 119-123. MR816893 (87h:55011)

25. Hatcher A.: Algebraic Topology, Cambridge University Press, Cambridge, 2002. MR.1867354 (2002k:55001)

26. Huebschmann J.: Crossed $n$-Fold Extensions of Groups and Cohomology, Comment. Math. Helv. 55 (1980), no. 2, 302-313. MR576608 (82e:20063)

27. Jajodia S.: On 2-Dimensional CW-Complexes with a Single 2-Cell, Pacific J. Math. 80 (1979), no. 1, 191-203. MR.534708 (80k:57005)

28. Kawauchi A., Shibuya T.T., Suzuki S.: Descriptions on Surfaces in Four-Space. I. Normal forms, Math. Sem. Notes Kobe Univ. 10 (1982) 75-125. MR672939 (84d:57017)

29. Kirby, Robion C.: The Topology of 4-Manifolds, Lecture Notes in Mathematics, 1374, Springer-Verlag, Berlin, 1989. MR1001966 (90j:57012)

30. Loday J.L.: Spaces with Finitely Many Nontrivial Homotopy Groups, J. Pure Appl. Algebra 24 (1982), no. 2, 179-202. MR651845 (83i:55009)

31. Lomonaco S.J. Jr.: The Homotopy Groups of Knots I. How to Compute the Algebraic 2-Type, Pacific J. Math. 95 (1981), no. 2, 349-390. MR632192 (83a:57025)

32. Mac Lane S.: Cohomology Theory in Abstract Groups III, Operator Homomorphisms of Kernels, Ann. of Math. (2) 50 (1949), 736-761. MR0033287 (11:415f)

33. Mac Lane S., Whitehead J.H.C.: On the 3-Type of a Complex, Proc. Nat. Acad. Sci. U. S. A. 36 (1950), 41-48. MR0033519(11:450h)

34. Matveev S.V.: The Structure of the Second Homotopy Group of the Join of Two Spaces. (Russian) Studies in topology, V. Zap. Nauchn. Sem. Leningrad. Otdel. Mat. Inst. Steklov. (LOMI) 143 (1985), 147-155, 178-179, Review in MathSciNet. MR806565 (87j:55016)

35. May J.P.: A Concise Course in Algebraic Topology, Chicago Lectures in Mathematics. University of Chicago Press, Chicago, IL, 1999. MR.1702278 (2000h:55002)

36. Mazur B.: Differential Topology From the Point of View of Simple Homotopy Theory, Inst. Hautes Études Sci. Publ. Math., no. 15, 1963. MR0161342 (28:4550)

37. Papakyriakopoulos C. D.: On Dehn's Lemma and the Asphericity of Knots, Ann. of Math. (2) 66 (1957), 1-26. MR0090053 (19:761a)

38. Plotnick S. P., Suciu A. I.: $k$-Invariants of Knotted 2-Spheres, Comment. Math. Helv. 60 (1985), no. 1, 54-84. MR787662 (86i:57026)

39. Porter T.: Interpretations of Yetter's Notion of $G$-Coloring: Simplicial Fibre Bundles and NonAbelian Cohomology, J. Knot Theory Ramifications 5 (1996), no. 5, 687-720. MR1414095 (97h:57030)

40. Porter T.: Topological Quantum Field Theories from Homotopy $n$-Types, J. London Math. Soc. (2) 58 (1998), no. 3, 723-732. MR1678163 (2000c:57064)

41. Rolfsen D.: Knots and Links. Mathematics Lecture Series, No. 7. Publish or Perish, Inc., Berkeley, Calif., 1976. MR 0515288 (58:24236)

42. Rourke C.P., Sanderson B.J.: Introduction to Piecewise-Linear Topology, Reprint, Springer Study Edition, Springer-Verlag, Berlin, New York, 1982. MR665919 (83g:57009)

43. Swenton F.J.: On a Calculus for 2-Knots and Surfaces in 4-Space, J. Knot Theory Ramifications 10 (2001), no. 8, 1133-1141. MR.1871221 (2002j:57043)

44. Whitehead G.W.: Elements of Homotopy Theory, Graduate Texts in Mathematics, 61, Springer-Verlag, New York, Berlin, 1978. MR.516508 (80b:55001)

45. Whitehead J.H.C.: On Adding Relations to Homotopy Groups, Ann. of Math. (2) 42 (1941), 409-428. MR0004123 (2:323c)

46. Whitehead J.H.C.: Note on a Previous Paper Entitled "On Adding Relations to Homotopy Groups", Ann. of Math. (2) 47 (1946), 806-810. MR0017537 (8:167a)

47. Whitehead J.H.C.: Combinatorial Homotopy. II, Bull. Amer. Math. Soc. 55 (1949), 453-496. MR.0030760 (11:48c) 
48. Yetter D.: TQFT's from Homotopy 2-Types, J. Knot Theory Ramifications 2 (1993), no. 1, 113-123. MR1209321 (94e:57028)

49. Yoshikawa K.: An Enumeration of Surfaces in Four-Space, Osaka J. Math. 31 (1994), 497-522. MR:1309400 (95m:57037)

Departamentos de Matemática, Centro de Matemática da Universidade do Porto, Rua do Campo Alegre, 687, 4169-007 Porto, Portugal

E-mail address: jnmartins@fc.up.pt 